\title{
The description of reflection coefficients of the scattering problems for finding solutions of the Korteweg-de Vries equations
}

Pham Loi Vu

To cite this article: Pham Loi Vu (2018) The description of reflection coefficients of the scattering problems for finding solutions of the Korteweg-de Vries equations, Journal of Nonlinear Mathematical Physics 25:3, 399-432, DOI:

https://doi.org/10.1080/14029251.2018.1494777

To link to this article: https://doi.org/10.1080/14029251.2018.1494777

Published online: 04 January 2021 


\title{
The description of reflection coefficients of the scattering problems for finding solutions of the Korteweg-de Vries equations
}

\author{
Pham Loi Vu \\ Institute of Mechanics, Vietnam Academy of Science and Technology, \\ 264 Doi Can Street, Hanoi, Vietnam \\ phamloivu77@gmail.com
}

Received 31 October 2017

Accepted 12 February 2018

\begin{abstract}
The results of inverse scattering problem associated with the initial-boundary value problem (IBVP) for the Korteweg-de Vries (KdV) equation with dominant surface tension are formulated. The necessary and sufficient conditions for given functions to be the left- and right-reflection coefficients of the scattering problem are established. The time-dependence $t, t>0$ of each element of the scattering matrix $s(k, t)$ is found in respective sector of the $k$-spectral plane by expansion formulas which are constructed from the known initial and boundary conditions of the IBVP. Knowing the right-reflection coefficient calculated from the elements of $s(k, t)$, we solve the Gelfand-Levitan-Marchenko (GLM) equation in the inverse problem. Then the solution of the IBVP is expressible through the solution of the GLM equation. The asymptotic behavior at infinity of time of the solution of the IBVP is shown
\end{abstract}

Keywords: Necessary and sufficient conditions; left- and right-reflection coefficients; time-evolution of scattering matrix; time-dependence of the reflection coefficients; soliton-solutions.

2000 Mathematics Subject Classification: 37K15, 35Q53

\section{Introduction}

After a series of papers by Fokas and Its [2], it became clear that under arbitrary boundary conditions solving the initial-boundary value problem (IBVP) for the nonlinear equations like the Korteweg-de Vries (KdV) or nonlinear Schrödinger (NLS) equations had not met the same success as solving the Cauchy problem for the KdV equation on the whole line. But there is a specific class of boundary conditions that are completely consistent with the integrability property. Under these conditions, the IBVPs are effectively embedded in the ISM schema. A number of examples of such boundary conditions were discussed in $[1,3,4,11,13]$. In the present paper we study inverse scattering problem (ISP) associated with the IBVP for the KdV equation:

$$
\begin{aligned}
& p_{t}-p_{x x x}+6 p p_{x}=0, \quad x>0, \quad t>0, \\
& p_{\mid x=0}=0, \quad p_{x x \mid x=0}=0, \\
& p_{\mid t=0}=p(x), \quad p(x)_{\mid x \rightarrow \infty} \rightarrow 0,
\end{aligned}
$$

where the boundary conditions (1.2) are consistent at the corner point, i.e., $p(0,0)=p_{x x}(0,0)=0$, and the function $p(x)$ that determines the initial condition (1.3) is required to satisfy the following conditions, which will be referred to as Conditions I:

Conditions I: The function $p(x)$ is real-valued infinitely smooth and tends to zero at infinity in the 
Schwartz sense, [10], i.e., $p(x)$ and all its derivatives decrease faster than any positive power of $x^{-1}$. At $x=0 p(x)$ vanishes together with all derivatives, and $\int_{0}^{\infty} p(x) \mathrm{d} x \neq 0$.

In [11] the problem of solving the considered IBVP is reduced to that of solving two ISPs. The first scattering problem (SP) is associated with the KdV equation (1.1), the second SP is selfconjugate.

In the present paper we prove the theorem of the necessary and sufficient conditions for given functions to be the left- and right-reflection coefficients of the first SP. The proof of this principal theorem is absent in $[1,3,4,11]$. Further, in Sec. 3 the scattering function of the self-conjugate SP is expressible through elements of the given scattering matrix $s(k)=s(k, 0)$ of the first SP. Knowing the scattering function, we solve the inverse SP for finding the unknown potential self-conjugate matrix. In Sec. 4 the time-dependence of elements of the matrix $s(k, t)$ is found in every respective sector of the $k$-spectral plane. The solution of the IBVP is expressible through the solution of the Galfand-Levitan-Marchenko (GLM) equation. The behaviour at infinity of $t$-time of the solution of the IBVP is shown. Exact soliton-solutions of the Cauchy problem for the KdV equation are presented in Sec. 5.

\section{The direct and inverse SP}

The IBVP (1.1)-(1.2)-(1.3) is associated with the SP on a half-line for a system of equations:

$$
\frac{\mathrm{d}}{\mathrm{d} x}\left(\begin{array}{c}
y \\
y_{x}
\end{array}\right)=\left(\begin{array}{cc}
0 & 1 \\
p(x)-\lambda & 0
\end{array}\right)\left(\begin{array}{c}
y \\
y_{x}
\end{array}\right), 0 \leq x<\infty
$$

with the boundary conditions as $x \rightarrow 0$ :

$$
\left(y(k, x), y_{x}(k, x)\right)=(1,-i k)+o(1), \lambda=k^{2} .
$$

The SP for the Schrödinger equation on the whole line is well studied, therefore it is convenient to reduce the SP (2.1)-(2.2) on the half-line to a problem on the whole line by continuing the potential $p(x)$ from the positive half-line to the whole line. The potential is continued trivially by setting $p(x) \equiv 0$ for all $x<0$. According to this way, we write system (2.1) in the form:

$$
y_{x}=U y, \quad U=\left(\begin{array}{cr}
0 & 1 \\
p-\lambda & 0
\end{array}\right), y=\left(y_{1}(k, x), y_{2}(k, x)\right), \lambda=k^{2},-\infty<x<\infty,
$$

where the function $p(x)$ satisfies Condition I, $p(x) \equiv 0$ for $x<0, y_{2}=y_{1 x}$ and $y_{1}$ satisfies the Schrödinger equation:

$$
-y_{x x}+p(x) y=k^{2} y,-\infty<x<\infty .
$$

The formulas (2.5)-(2.23) presented below are deduced from the known facts of the scattering theory for equation (2.4) (see [5,7]). We construct the matrix solutions of system (2.1):

$$
E=\left(E^{+}, E^{-}\right)=\left(\begin{array}{cc}
e(k, x) & e(-k, x) \\
e_{x}(k, x) & e_{x}(-k, x)
\end{array}\right), \quad W=\left(W^{-}, W^{+}\right)=\left(\begin{array}{cc}
\omega(-k, x) & \omega(k, x) \\
\omega_{x}(-k, x) & \omega_{x}(k, x)
\end{array}\right)
$$


with the conditions for all real $k=\sqrt{\lambda}$ :

$$
\begin{aligned}
& E=T(k)\left(\begin{array}{cc}
e^{i k x} & 0 \\
0 & e^{-i k x}
\end{array}\right)+o(1) \quad(x \rightarrow \infty), \\
& W=T(k)+o(1) \quad(x \rightarrow 0), \quad T(k)=\left(\begin{array}{cc}
1 & 1 \\
i k-i k
\end{array}\right) .
\end{aligned}
$$

The function $e(k, x)$ satisfies the integral equation:

$$
e(k, x)=e^{i k x}+\int_{x}^{\infty} \frac{\sin k(s-x)}{k} p(s) e(k, s) \mathrm{d} s,
$$

and can be represented as:

$$
e(k, x)=e^{i k x}+\int_{x}^{\infty} K(x, \xi) e^{i k \xi} \mathrm{d} \xi, \operatorname{Im} k \geq 0,
$$

where the function $K(x, \xi)$ is the kernel of the transformation operator and is determined from the integral equation of the Volterra type:

$$
K(x, \xi)=\frac{1}{2} \int_{\frac{x+\xi}{2}}^{\infty} p(s) \mathrm{d} s+\int_{\frac{x+\xi}{2}}^{\infty} \mathrm{d} \alpha \int_{0}^{\frac{\xi-x}{2}} p(\alpha-\beta) K(\alpha-\beta, \alpha+\beta) \mathrm{d} \beta,
$$

with $K(x, \xi)=0$ for $\xi<x$. Consequently, $K(x, \xi)$ satisfies the estimate:

$$
|K(x, \xi)| \leq C \int_{\frac{x+\xi}{2}}^{\infty}|p(s)| \mathrm{d} s,
$$

and the conditions:

$$
-2 \frac{\mathrm{d}}{\mathrm{d} x} K(x, x)=p(x), \quad\left(\frac{\mathrm{d}}{\mathrm{d} x} K(x, x)\right)_{x=0}=-\frac{1}{2} p(0)=0 .
$$

The function $\omega(k, x)$ satisfies the integral equation:

$$
\omega(k, x)=e^{-i k x}+\int_{0}^{x} \frac{\sin k(x-s)}{k} p(s) \omega(k, s) \mathrm{d} s, \operatorname{Im} k \geq 0,
$$

and can be represented as:

$$
\omega(k, x)=\left\{\begin{array}{l}
e^{-i k x}+\int_{-\infty}^{x} K^{-}(x, \xi) e^{-i k \xi} \mathrm{d} \xi \text { for } x>0, \\
e^{-i k x} \text { for } x \leq 0
\end{array}\right.
$$

where $K^{-}(x, \xi) \equiv 0$ for $\xi \leq x \leq 0$, and $2 \frac{\mathrm{d}}{\mathrm{d} x} K^{-}(x, x)=\left\{\begin{array}{l}p(x) \text { for } x>0 \\ 0 \text { for } x \leq 0\end{array}\right.$

In view of the reality of $p(x)$, the functions $K(x, \xi)$ and $K^{-}(x, \xi)$ are real-valued and therefore

$$
e(k, x)=\overline{e(-k, x)}, e_{x}(k, x)=\overline{e_{x}(-k, x)}, \omega(k, x)=\overline{\omega(-k, x)}, \omega_{x}(k, x)=\overline{\omega_{x}(-k, x)} .
$$

The solutions $e(k, x)$ and $\omega(k, x)$ of system (2.3) admit from the real line an analytical continuation into the upper half-plane $\operatorname{Im} k>0$. Since the Wronskians of the solutions do not depend on $x$, then

$$
\begin{aligned}
& W\{\omega(-k, x), \omega(k, x)\}=W\{e(k, x), e(-k, x)\} \\
& =e(k, x) e_{x}(-k, x)-e_{x}(k, x) e(-k, x)=-2 i k \text { for real } k \neq 0 .
\end{aligned}
$$


Hence, $(e(k, x), e(-k, x))$ and $(\omega(-k, x), \omega(k, x))$ for real $k \neq 0$ are bases of solutions of system (2.3), therefore

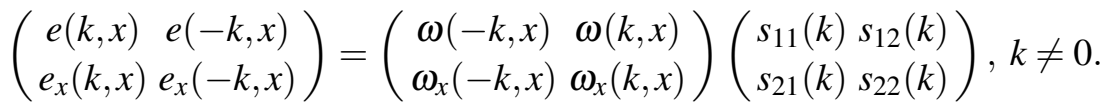

$$
\begin{aligned}
& \left(\begin{array}{cc}
\omega(-k, x) & \omega(k, x) \\
\omega_{x}(-k, x) & \omega_{x}(k, x)
\end{array}\right)=\left(\begin{array}{cc}
e(k, x) & e(-k, x) \\
e_{x}(k, x) & e_{x}(-k, x)
\end{array}\right)\left(\begin{array}{cc}
s_{11}(-k) & -s_{21}(-k) \\
-s_{21}(k) & s_{11}(k)
\end{array}\right), k \neq 0 .
\end{aligned}
$$

The matrix $s(k)=\left(\begin{array}{l}s_{11}(k) s_{12}(k) \\ s_{21}(k) s_{22}(k)\end{array}\right)$ thus defined is called the scattering matrix of the SP (2.3), (2.6). Using (2.6), (2.14), we determine the entries $s_{i j}(k)$ of $s(k)$ in terms of boundary values (BVs) $\left(e(k), e_{x}(k)\right)=\left(e(k, 0), e_{x}(k, 0)\right)$ for $k \neq 0$ :

$$
\begin{aligned}
s_{11}(k) & =\frac{1}{2} e(k)+\frac{1}{2 i k} e_{x}(k), & s_{12}(k) & =\frac{1}{2} e(-k)+\frac{1}{2 i k} e_{x}(-k), \\
s_{21}(k) & =\frac{1}{2} e(k)-\frac{1}{2 i k} e_{x}(k), & s_{22}(k) & =\frac{1}{2} e(-k)-\frac{1}{2 i k} e_{x}(-k),
\end{aligned}
$$

where due to (2.6) and (2.13) the BVs $\left(e(k), e_{x}(k)\right)$ are different from zero for all real $k \neq 0$.

Remark $2.1([5,7])$. For real values of the parameter $k$ the entries $s_{i j}(k)$ of $s(k)$ possess the properties:

1. The involutions: $s_{11}(-k)=\overline{s_{11}(k)}, s_{21}(-k)=\overline{s_{21}(k)}, s_{22}(k)=s_{11}(-k), s_{12}(k)=s_{21}(-k)$.

2. The constraint: $\operatorname{det} s(k)=1=\left|s_{11}(k)\right|^{2}-\left|s_{12}(k)\right|^{2},|t(k)|^{2}+|\tilde{r}(k)|^{2}=1, t(k)=s_{11}^{-1}(k)$,

$$
\tilde{r}(k)=\frac{s_{21}(k)}{s_{11}(k)}, \quad \tilde{R}(k)=-\frac{s_{12}(k)}{s_{11}(k)}=-\frac{s_{11}(-k)}{s_{11}(k)} \tilde{r}(-k), \quad s_{11}(k) \neq 0 .
$$

The functions $s_{11}(k)$ and $s_{21}(k)$ are called the refraction and reflection coefficients, respectively. The functions $\tilde{R}(k)$ and $\tilde{r}(k)$ are called the right- and left-reflection coefficients, respectively for the waves incident on the potential $p(x)$ from the right.

Substituting (2.7) for $x=0$ into (2.16) with due regard for (2.8), we obtain:

$$
\begin{aligned}
& \begin{aligned}
2 i k\left(s_{11}(k)-1\right) & =2 i k\left(s_{22}(-k)-1\right)=-\int_{0}^{\infty} e^{-i k s} p(s) e(k, s) \mathrm{d} s \\
& =-\int_{0}^{\infty} p(\xi) \mathrm{d} \xi-\int_{0}^{\infty} e^{i k \xi} \mathrm{d} \xi \int_{0}^{\infty} p(s) K(s, \xi+s) \mathrm{d} s, \quad \operatorname{Im} k \geq 0
\end{aligned} \\
& 2 i k s_{21}(k)=2 i k s_{12}(-k)=\int_{0}^{\infty} e^{i k s} p(s) e(k, s) \mathrm{d} s \\
& =\frac{1}{2} \int_{0}^{\infty} e^{i k \xi} p\left(\frac{\xi}{2}\right) \mathrm{d} \xi+\int_{0}^{\infty} e^{i k \xi} \mathrm{d} \xi \int_{0}^{\frac{\xi}{2}} p(s) K(s, \xi-s) \mathrm{d} s, \quad \operatorname{Im} k \geq 0 .
\end{aligned}
$$

Owing to Conditions I and (2.10) the functions $s_{i j}(k)$ have the asymptotic behavior:

$$
\begin{aligned}
& s_{11}(k)-1=s_{22}(-k)-1=O\left(\frac{1}{|k|}\right) \text { as }|k| \rightarrow \infty, \\
& s_{21}(k)=s_{12}(-k)=o\left(\frac{1}{|k|}\right) \text { as }|k| \rightarrow \infty .
\end{aligned}
$$


The integral representations for $s_{i j}(k)$ are obtained from (2.17) and (2.18):

$$
\begin{aligned}
& 2 i k s_{12}(-k)=2 i k s_{21}(k)=\int_{0}^{\infty} e^{i k \xi} A(\xi) \mathrm{d} \xi \operatorname{Im} k \geq 0, \\
& 2 i k\left(s_{11}(k)-1\right)=2 i k\left(s_{22}(-k)-1\right)=-\int_{0}^{\infty} p(\xi) \mathrm{d} \xi-\int_{0}^{\infty} e^{i k \xi} B(\xi) \mathrm{d} \xi \operatorname{Im} k \geq 0,
\end{aligned}
$$

where

$$
A(\xi)=\frac{1}{2} p\left(\frac{\xi}{2}\right)+\int_{0}^{\frac{\xi}{2}} p(s) K(s, \xi-s) \mathrm{d} s, B(\xi)=\int_{0}^{\infty} p(s) K(s, \xi+s) \mathrm{d} s .
$$

Lemma 2.1. The coefficients $s_{21}(k)$ and $s_{11}(k)$ of the scattering matrix $s(k)$ of the $S P(2.3),(2.6)$ are infinitely differentiable for functions $k \neq 0, \operatorname{Im} k \geq 0$. Their derivatives satisfy the estimates as $k \rightarrow \infty$ :

$$
\begin{aligned}
& \left|s_{21}^{(m)}(k)\right| \leq C_{m, j}|k|^{-j}, \quad m, j=0,1,2, \ldots \\
& \left|s_{11}(k)-1\right| \leq C|k|^{-1}, \quad\left|s_{11}^{(m)}(k)\right| \leq C_{m}|k|^{-1}, \quad m=1,2, \ldots
\end{aligned}
$$

The functions $2 i k s_{11}(k)$ and $2 i k s_{21}(k)$ are continuous in the closed half-plane $\operatorname{Im} k \geq 0$.

Proof. Owing to Conditions I and (2.10) of the potential $p$, the function $K(x, \xi)$ from Eq. (2.9) is real-valued and infinitely differentiable with respect to each variable of $x$ and $\xi$. Furthermore, $K(x, \xi)$ and all its derivatives decrease faster than any positive power of $x^{-1}$ and $\xi^{-1}$. Therefore, the functions $A(\xi)$ and $B(\xi)$ defined by (2.23) are infinitely differentiable and decrease faster than any positive power of $\xi^{-1}$ :

$$
|A(\xi)| \leq C_{A}|\xi|^{-j},|B(\xi)| \leq C_{B}|\xi|^{-j}, \quad j=0,1,2, \ldots
$$

Using Condition I of $p$ and the smoothness and estimates of $K$, from (2.23) we get:

$$
\left|A^{(m)}(\xi)\right| \leq C_{A m}|\xi|^{-j}, m, j=0,1,2, \ldots
$$

Since $p(0)=0$, then $A(0)=0$. It can be proved by induction that

$$
\left|A^{(m)}(\xi)\right|_{\xi=0}=0, \quad m=1,2, \ldots
$$

Due to (2.26) the functions $s_{21}(k)$ and $s_{11}(k)$ defined by (2.21) and (2.22), respectively are infinitely differentiable for $k \neq 0$. Then, from (2.21)-(2.23) it follows that the functions $2 i k s_{11}(k)$ and $2 i k s_{21}(k)$ are continuous in the closed half-plane $\operatorname{Im} k \geq 0$. The estimate (2.24) can be proved by induction with respect to $m$. Indeed, by using equality (2.26)-(2.28) and integrating the Fourier integral (2.21) by parts $j$ times, we get:

$$
2 i k(i k)^{j} s_{21}(k)=\int_{0}^{\infty} e^{i k \xi} A^{(j)}(\xi) \mathrm{d} \xi
$$

whence it follows that $\left|s_{21}(k)\right| \leq C_{0, j}|k|^{-j}, j=0,1,2, \ldots$ and $m=0$. Thus, the estimate (2.24) is true for $j=0,1,2, \ldots$ and $m=0$. Supposing that the estimate (2.24) is proved for $m-1$ :

$$
\left|s_{21}^{(m-1)}(k)\right| \leq C_{m-1, j}|k|^{-j}
$$


Differentiating (2.21) $m$ times and using the Leibniz's Rule, we obtain:

$$
\left(2 i k s_{21}(k)\right)^{(m)}=2 i k s_{21}^{(m)}(k)+2 i m s_{21}^{(m-1)}(k)=\int_{0}^{\infty} e^{i k \xi}(i \xi)^{m} A(\xi) \mathrm{d} \xi .
$$

Integrating the integral in the right-hand side of (2.29) by parts $j-1$ times, using (2.20), (2.26)(2.28), we calculate:

$$
\begin{aligned}
& \int_{0}^{\infty} e^{i k \xi}(i \xi)^{m} A(\xi) \mathrm{d} \xi=\frac{1}{i k} e^{i k \xi}\left[(i \xi)^{m} A(\xi)\right]_{\xi=0, \infty}-\frac{1}{i k} \int_{0}^{\infty} e^{i k \xi}\left[(i \xi)^{m} A(\xi)\right]^{\prime} \mathrm{d} \xi=\cdots \\
& =\frac{1}{(i k)^{j-1}} e^{i k \xi}\left[(i \xi)^{m} A(\xi)\right]_{\xi=0, \infty}^{(j-2)}-\frac{1}{(i k)^{j-1}} \int_{0}^{\infty} e^{i k \xi}\left[(i \xi)^{m} A(\xi)\right]^{(j-1)} \mathrm{d} \xi \\
& =-\frac{1}{(i k)^{j-1}} \int_{0}^{\infty} e^{i k \xi}\left[(i \xi)^{m} A(\xi)\right]^{(j-1)} \mathrm{d} \xi .
\end{aligned}
$$

Using the right-hand side of the above equality and the induction hypothesis for $s_{21}^{(m-1)}(k)$, from (2.29) we obtain the estimate (2.24) for $s_{21}^{(m)}(k)$.

The first inequality of (2.25) is deduced from estimate (2.19). We prove the second inequality of (2.25). Differentiating (2.22) $m$ times, yield:

$$
\left(2 i k s_{11}(k)\right)^{(m)}=2 i k s_{11}^{(m)}(k)+2 i m s_{11}^{(m-1)}(k)=-\int_{0}^{\infty} e^{i k \xi}(i \xi)^{m} B(\xi) \mathrm{d} \xi .
$$

Using (2.26) and the induction hypothesis for $s_{11}^{(m-1)}(k)$, from the latest equality we obtain the second estimate of (2.25). The number $B(0)$ is nonzero. Indeed, using (2.9) and Conditions I of $p$, we calculate:

$$
\begin{array}{r}
B(0)=\int_{0}^{\infty} p(s) K(s, s) \mathrm{d} s=\frac{1}{2} \int_{0}^{\infty} p(s) \mathrm{d} s \int_{s}^{\infty} p(\xi) \mathrm{d} \xi=\frac{1}{2}\left\{\int_{0}^{\infty} p(s) \mathrm{d} s\right\}^{2}- \\
\frac{1}{2} \int_{0}^{\infty} p(s) \mathrm{d} s \int_{0}^{s} p(\xi) \mathrm{d} \xi=\frac{1}{2}\left\{\int_{0}^{\infty} p(s) \mathrm{d} s\right\}^{2}-B(0) .
\end{array}
$$

Hence, $B(0)=\frac{1}{4}\left\{\int_{0}^{\infty} p(s) d s\right\}^{2} \neq 0$. On account of this fact, the function $s_{11}^{(m)}(k)$ obeys the estimate (2.25) as the first power of $k^{-1}$. Lemma 2.1 is proved.

The following remark is deduced from Lemma 2.1, Remark 2.1 and properties of the solutions of problems (2.3), (2.5) and (2.3), (2.6).

\section{Remark 2.2 ([5,7]).}

1. The analytic continuation of the function $s_{11}(k)$ with respect to $k$ from the real axis into the upper half-plane $\operatorname{Im} k>0$ can have a finite number of simple zeros on the positive imaginary axis at $k_{j}=i \mu_{j}, \mu_{j}>0, j=1, \ldots, N$;

2. There is a one-to-one correspondence between the simple pole $i \mu_{j}, \mu_{j}>0$ of $\tilde{r}(k) \operatorname{Im} k>0$ and the simple negative eigenvalues $-\mu_{j}^{2}, j=1, \ldots, N$ of the $\mathrm{SP}(2.1)-(2.2)$. 
Suppose that the potential function $p(x)$ is to be subjected to the following restriction, which will be referred to as the condition II.

Condition II. The potential function $p(x)$ is to be subjected to the condition that Eq. (2.4) must not have a discrete spectrum. Then by Remark 2.2, $s_{11}(k) \neq 0$ for all $k, \operatorname{Im} k>0$.

The scattering matrix $s(k)$ gives complete information about the continuous spectrum of the Schrödinger operator. By Condition II, Remark 2.2 and the dispersion relation, we can show that essentially, all information about $s(k)$ is contained in the right-reflection coefficient $\tilde{R}(k)$. Denote by $\mathscr{S}$ the class of all real-valued functions satisfying Conditions I and II. From (2.14) and (2.15) it follows that the functions:

$$
\begin{aligned}
& {\left[s_{11}(k)\right]^{-1} e(k, x)=\omega(-k, x)+\tilde{r}(k) \omega(k, x),} \\
& {\left[s_{11}(k)\right]^{-1} \omega(k, x)=e(-k, x)+\tilde{R}(k) e(k, x),}
\end{aligned}
$$

are bounded solutions of system (2.1) with the potential $p(x)$ belonging to the class $\mathscr{S}$. The behaviour of the function $k s_{11}(k)$ as $k \rightarrow 0$ is closely related to that of functions $\tilde{R}(k)$ and $\tilde{r}(k)$ as $k \rightarrow 0$. In fact, upon rewriting (2.30) and (2.31) and letting $k \rightarrow 0$, gives:

$$
\begin{gathered}
0=e(0, x) \lim _{k \rightarrow 0} k s_{11}(k)\{\tilde{R}(k)+1\}=\omega(0, x) \lim _{k \rightarrow 0} k s_{11}(k)\{\tilde{r}(k)+1\} \text {. Consequently, } \\
\lim _{k \rightarrow 0} k s_{11}(k)\{\tilde{R}(k)+1\}=\lim _{k \rightarrow 0} k s_{11}(k)\{\tilde{r}(k)+1\}=0 .
\end{gathered}
$$

From condition (2.32) we have the estimate: $k\left\{s_{11}(k)+s_{21}(k)\right\}=o(1)$ as $k \rightarrow 0$.

To fulfill this estimate, the following condition must be satisfied:

$$
s_{11}(k)+s_{21}(k)=O(1) \text { as } k \rightarrow 0,
$$

Conversely, if the condition (2.33) is fulfilled, then the condition (2.32) is satisfied.

Lemma 2.2. The left-reflection coefficient $\tilde{r}(k)$ of the SP for system (2.1) with the potential $p(x)$ belonging to the class $\mathscr{S}$ and boundary condition (2.2) obeys the following conditions:

1. For all $k, \operatorname{Im} k \geq 0$, the function $\tilde{r}(k)$ is completely continuous and infinitely differentiable. $\tilde{r}(k)$ and all its derivatives decrease faster than any positive power of $k^{-1}$, and

$$
\overline{\tilde{r}(k)}=\tilde{r}(-k), \quad|\tilde{r}(k)|<1 \text { for real } k \neq 0 .
$$

If the residues of the functions $s_{11}(k)$ and $s_{21}(k)$ at $k=0$ are different from zero, then $\tilde{r}(0)=-1$.

2. The function $\tilde{r}(k)$ admits the Fourier integral representation:

$$
\tilde{r}(k)=\int_{0}^{\infty} e^{i k x} r(x) \mathrm{d} x \text { for all } k, \operatorname{Im} k \geq 0,
$$

where $r(x)$ is a completely continuous and rapidly decreasing function, which is defined by the inverse Fourier transform:

$$
r(x)=\frac{1}{2 \pi} \int_{-\infty}^{\infty} e^{-i k x} \tilde{r}(k) \mathrm{d} k, \text { for } x>0 .
$$

The function $r(x)$ is infinitely differentiable:

$$
r^{(m)}(x)=\frac{1}{2 \pi} \int_{-\infty}^{\infty}(-i k)^{m} e^{-i k x} \tilde{r}(k) \mathrm{d} k \text { for } x>0, m=1,2, \ldots
$$

In addition $r(x)$ and $r^{(m)}(x)$ are real-valued functions vanishing at $x=0$. 
Proof. The validity of assertion 1 for $k \neq 0, \operatorname{Im} k \geq 0$ follows from Lemma 2.1 and Remarks 2.1, 2.2. We prove the smoothness of $\tilde{r}(k)$ and its derivatives at $k=0$. From (2.16) we have the relations:

$$
2 i k s_{21}(k)=i k e(k)-e_{x}(k), \quad 2 i k s_{11}(k)=i k e(k)+e_{x}(k) .
$$

These relations make clear that the entries $s_{i j}(k), i, j=1,2$ of $s(k)$ have generally a simple pole at $k=0$. The residues of functions $2 i k s_{11}(k)$ and $2 i k s_{21}(k)$ at $k=0$ are defined by:

$$
\operatorname{Res}\left[2 i k s_{11}(k), 0\right]=-\operatorname{Res}\left[2 i k s_{21}(k), 0\right]=e_{x}(0) .
$$

If $e_{x}(0) \neq 0$, then the function $\tilde{r}(k)$ and its derivatives are continuous and smooth at $k=0$ :

$$
\lim _{k \rightarrow 0} \tilde{r}(k)=\lim _{k \rightarrow 0} \frac{2 i k s_{21}(k)}{2 i k s_{11}(k)}=\lim _{k \rightarrow 0} \frac{-e_{x}(k)}{e_{x}(k)}=\frac{-e_{x}(0)}{e_{x}(0)}=-1
$$

If $e_{x}(0)=0$, i.e., the residues (2.37) are zero, then the functions $s_{11}(k)$ and $s_{21}(k)$ are continuous and analytic at $k=0$. The constraint condition $\left|s_{11}(k)\right|^{2}-\left|s_{21}(k)\right|^{2}=1$ implies that $\left|s_{11}(k)\right|>1$ for $k \in \mathbb{R}$. Therefore, the function $\tilde{r}(k)$ and its derivatives are continuous and smooth at $k=0$.

By Lemma 2.1 the functions $\tilde{r}(k)$ and $(-i k)^{m} \tilde{r}(k)$ are analytic in the upper half-plane $\operatorname{Im} k \geq 0$ and rapidly decrease at infinity for any nonnegative integer $m$. On account of this fact, the function $\tilde{r}(k)$ admits the Fourier integral representation (2.35) of a function $r(x)$ for $x>0$. Since the Fourier transform (2.35) and its inverse Fourier transform (2.36) maps $\mathscr{S}$ onto $\mathscr{S}$ mutually continuously one-to-one [10], then $r(x)$ and its derivatives $r^{(m)}(x)$ for $x>0$ defined by (2.36) are rapidly decreasing functions. Due to (2.34) these functions are real-valued. We have from (2.36):

$$
r^{(m)}(0)=\frac{1}{2 \pi} \int_{-\infty}^{\infty}(-i k)^{m} \tilde{r}(k) \mathrm{d} k ; m=0,1,2, \ldots
$$

where the integrand function $G(k)=(-i k)^{m} \tilde{r}(k)$ and its derivatives $G^{(j)}(k)$ are analytic in the upper half-plane $\operatorname{Im} k \geq 0$ and rapidly decrease at infinity for nonnegative integer $m$, and $G(\infty)=$ $G^{(j)}(\infty)=0, j=1,2, \ldots$ Hence, the function $G(k)$ is analytic in the closed upper half-plane $\operatorname{Im} k \geq 0$, therefore, by the Cauchy's Theorem the right-hand side of the last formula vanishes, i.e., $r^{(m)}(0)=0, m=0,1,2, \ldots$ Lemma 2.2 is proved.

The further conditions of $\tilde{R}(k)$ are deduced from Lemma 2.1, Remarks 2.1 and 2.2.

Lemma 2.3. The right reflection coefficient $\tilde{R}(k)$ of the SP for system (2.1) with the potential $p(x)$ belonging to the class $\mathscr{S}$ and boundary condition (2.2) obeys the following conditions:

1. The function $\tilde{R}(k)$ is completely continuous and infinitely differentiable for all real $k \in(-\infty, \infty)$. $\tilde{R}(k)$ and all its derivatives decrease faster than any positive power of $k^{-1}$, and

$$
\overline{\tilde{R}(k)}=\tilde{R}(-k), \quad|\tilde{R}(k)|=|\tilde{r}(k)|<1 \text { for real } k \neq 0
$$

If the residues of the functions $s_{11}(k)$ and $s_{12}(k)$ at $k=0$ are different from zero, then $\tilde{R}(0)=-1$; 
2. For real $k$ the function $\tilde{R}(k)$ admits the Fourier transform:

$$
\tilde{R}(k)=\int_{-\infty}^{\infty} e^{-i k x} R(x) \mathrm{d} x,
$$

where $R(x)$ is a real completely continuous and rapidly decreasing function, which is defined by the inverse Fourier transform:

$$
R(x)=\frac{1}{2 \pi} \int_{-\infty}^{\infty} e^{i k x} \tilde{R}(k) \mathrm{d} k
$$

The function $R(x)$ is infinitely differentiable:

$$
R^{(m)}(x)=\frac{1}{2 \pi} \int_{-\infty}^{\infty}(i k)^{m} e^{i k x} \tilde{R}(k) \mathrm{d} k, m=1,2, \ldots
$$

where the Fourier transform (2.39) and its inverse Fourier transform (2.40) maps $\mathscr{S}$ onto $\mathscr{S}$ mutually continuously one-to-one, [10]. Due to this fact and (2.38) $R(x)$ and $R^{(m)}(x), m=1,2, \ldots$ are rapidly decreasing and real-valued functions.

To recover the SP (2.1)-(2.2) from the right-reflection coefficient $\tilde{R}(k)$, we derive the fundamental integral equation connecting the given $\tilde{R}(k)$ with the kernels of the transformation operator. The following equation is derived from $(2.31)$ (see $[5,7])$ :

$$
R(x+y)+K(x, y)+\int_{x}^{\infty} R(y+\xi) K(x, \xi) \mathrm{d} \xi=0 \text { for } y>x,
$$

which is the Gelfand-Levitan-Machenko equation in the case of a purely continuous spectrum. In Eq. (2.41) $x$ is a parameter, $R(x+y)$ is a known function satisfying conditions enumerated in Lemma 2.3, and $K(x, y)$ is an unknown function of $y$ for every $x \in[0, \infty)$. Owing to conditions (2.38)-(2.40) of the function $R(x+y)$, Eq. (2.41) has a unique solution $K(x, y)$ either in $L_{1}[x, \infty)$ or $L_{2}[x, \infty)$.

Analogously, the following fundamental integral equation is derived from (2.30):

$$
r(x+y)+K^{-}(x, y)+\int_{-\infty}^{x} r(y+\xi) K^{-}(x, \xi) \mathrm{d} \xi=0 \text { for } y<x
$$

Owing to conditions (2.34)-(2.36) of the known function $r(x+y)$ Eq. (2.42) has a unique solution in either $L_{1}(-\infty, x]$ or $L_{2}(-\infty, x]$.

We use Eq. (2.41) to extract information on the solution $K(x, y), y>x$ from the conditions of the known function $R(x)$ in this equation. In fact, the function $K(x, y), y>x$ satisfies conditions, which are analogous to the conditions of the function $R(x)$. As has been proved in [5,7] that the solution $K(x, y)$ of Eq. (2.41) is the kernel of the transformation operator and the function constructed from $K(x, y)$ :

$$
e(k, x)=e^{i k x}+\int_{x}^{\infty} K(x, \xi) e^{i k \xi} \mathrm{d} \xi, \operatorname{Im} k \geq 0
$$

satisfies the Schrödinger equation (2.4):

$$
-e^{\prime \prime}(k, x)+p(x) e(k, x)=k^{2} e(k, x)
$$


with the potential $p(x)$ constructed from the solution of Eq. (2.41) by the formula:

$$
p(x)=-2 \frac{\mathrm{d}}{\mathrm{d} x} K(x, x) \text { for } x>0 .
$$

Substituting (2.43) into (2.7), we obtain integral Eq. (2.9) with the constructed potential (2.45). Since the solution of (2.9) is unique, then $K(x, y)$ satisfies conditions (2.11).

By an argument analogous to the previous one, we can prove that the function constructed from the solution $K^{-}(x, y)$ of Eq. (2.42):

$$
\omega(k, x)=e^{-i k x}+\int_{-\infty}^{x} K^{-}(x, \xi) e^{-i k \xi} \mathrm{d} \xi, \operatorname{Im} k \geq 0,
$$

satisfies the Schrödinger equation (2.4):

$$
-\omega^{\prime \prime}(k, x)+p^{-}(x) \omega(k, x)=k^{2} \omega(k, x)
$$

with the potential $p^{-}(x)$ constructed by $p^{-}(x)=2 \frac{\mathrm{d}}{\mathrm{d} x} K^{-}(x, x)$ for $x>0$.

The principal mathematical problem in inverse problem consists in the proof of the fact that under conditions (properties) of functions $s_{11}(k), s_{21}(k)$ enumerated in Remark 2.1 and Lemma 2.1, the procedure actually leads to the same differential equation, i.e., $p^{-}(x) \equiv p(x)$. This leads to describing the scattering data, i.e., to establishing the necessary and sufficient conditions of functions $\tilde{r}(k)$ and $\tilde{R}(k)$ to be the left- and right-reflection coefficients of the considered problem.

Theorem 2.1. Suppose that the functions $s_{11}(k)$ and $s_{21}(k),-\infty<k<\infty$ satisfy the conditions enumerated in Remark 2.1, Lemma 2.1, condition (2.33) and the function $s_{11}(k)$ admits an analytical continuation into the upper half-plane $\operatorname{Im} k>0$ and has no zeros there. Then

1. The functions $e(k, x)$ and $\omega(k, x)$ constructed from the solutions $K(x, y) \in L_{j}[x, \infty)$ and $K^{-}(x, y) \in$ $L_{j}(-\infty, x], j=1,2$ of Eqs. (2.41) and (2.42), respectively satisfy the same Shrödinger equation (2.4) with the constructed potential:

$$
\begin{aligned}
& p^{-}(x) \equiv p(x)=-2 \frac{\mathrm{d}}{\mathrm{d} x} K(x, x), \\
& p(0)=-2 \frac{\mathrm{d}}{\mathrm{d} x}(K(x, x))_{x=0}=0 .
\end{aligned}
$$

2. The conditions of functions $s_{11}(k)$ and $s_{21}(k)$ are both necessary and sufficient for the ratios of the type:

$$
\tilde{r}(k)=\frac{s_{21}(k)}{s_{11}(k)} . \operatorname{Im} k \geq 0 \text { and } \tilde{R}(k)=-\frac{s_{12}(k)}{s_{11}(k)},-\infty<k<\infty
$$

to be the left-reflection and right-reflection coefficients of the SP for one and the same system (2.1) with boundary condition (2.2) and constructed potential (2.48) belonging to the class $\mathscr{S}$. The Schrödinger equation (2.4) is restored precisely from $\tilde{R}(k)$.

Proof. The functions given by (2.43) and (2.46) admit analytic continuations into the upper halfplane $\operatorname{Im} k>0$. Extend the domain of the function $K^{-}(x, y)$ by setting: $K^{-}(x, y)=0$ for $y>x$. 
Further, we put:

$$
\tilde{\Phi}(x, y)=K^{-}(x, y)+r(x+y)+\int_{-\infty}^{x} r(y+\xi) K^{-}(x, \xi) \mathrm{d} \xi \text { for all real } y .
$$

Due to Eq. (2.42), $\tilde{\Phi}(x, y)=0$ for $y<x$. For $y>x$ :

$$
\tilde{\Phi}(x, y)=r(x+y)+\int_{-\infty}^{x} r(y+\xi) K^{-}(x, \xi) \mathrm{d} \xi, \quad \int_{-\infty}^{\infty}|\tilde{\Phi}(x, y)| \mathrm{d} y=\int_{x}^{\infty}|\tilde{\Phi}(x, y)| \mathrm{d} y<\infty .
$$

Multiply both sides of equality (2.50) by $e^{i k y}$, then integrate with respect to $y$ and apply the inverse Fourier formula to $r(x+y)$ :

$$
\int_{x}^{\infty} \tilde{\Phi}(x, y) e^{i k y} \mathrm{~d} y=\int_{-\infty}^{x} K^{-}(x, y) e^{i k y} \mathrm{~d} y+\frac{s_{21}(k)}{s_{11}(k)} e^{-i k x}+\frac{s_{21}(k)}{s_{11}(k)} \int_{-\infty}^{x} K^{-}(x, \xi) e^{-i k \xi} \mathrm{d} \xi .
$$

Adding $e^{i k x}$ to the right- and left- hand sides of the last equality and using (2.46), gives:

$$
e^{i k x}+\int_{x}^{\infty} \tilde{\Phi}(x, y) e^{i k y} \mathrm{~d} y=\omega(-k, x)+\frac{s_{21}(k)}{s_{11}(k)} \omega(k, x) .
$$

Multiply (2.51) by $s_{11}(k)$, then

$$
\begin{aligned}
& s_{11}(k) \omega(-k, x)+s_{21}(k) \omega(k, x)=e^{*}(k, x), \\
& e^{*}(k, x)=s_{11}(k)\left\{e^{i k x}+\int_{x}^{\infty} \tilde{\Phi}(x, y) e^{i k y} \mathrm{~d} y\right\} .
\end{aligned}
$$

Replacing $k$ by $-k$ in (2.52), yields:

$$
s_{11}(-k) \omega(k, x)+s_{21}(-k) \omega(-k, x)=e^{*}(-k, x) .
$$

Solving the system (2.52), (2.54) for $\omega(k, x)$, using conditions of $s_{11}(k)$ and $s_{21}(k)$, gives:

$$
\omega(k, x)=s_{11}(k) e^{*}(-k, x)-s_{21}(-k) e^{*}(k, x)
$$

In order to prove the identity (2.48), we need to prove that

$$
e^{*}(k, x) \equiv e(k, x)
$$

Indeed, if identity (2.56) will be proved, then due to (2.15) and (2.44), it follows from (2.55):

$$
-\omega^{\prime \prime}(k, x)=-s_{11}(k) e^{\prime \prime}(-k, x)+s_{21}(-k) e^{\prime \prime}(k, x)=\left[k^{2}-p(x)\right] \omega(k, x),
$$

because of (2.47), we obtain identity (2.48).

To prove identity (2.56), certain properties of the function $e^{*}(k, x)$ should be established.

a./ The function $e^{*}(k, x)$ defined by formula (2.53) admits an analytic continuation into the upper half-plane $\operatorname{Im} k>0$. Using estimate (2.25) for large $k$, from (2.53) we obtain the following estimate:

$$
\left|e^{*}(k, x)-e^{i k x}\right|=O\left(\frac{e^{-x \mathrm{Imk}}}{|k|}\right) \text { as } k \rightarrow \infty .
$$


b./ The function $k e^{*}(k, x)$ is continuous in the closed upper half-plane $\operatorname{Im} k \geq 0$ and in the neighbourhood of the point $k=0$, this function satisfies uniformly the estimate:

$$
k e^{*}(k, x)=o(1) \text { as } k \rightarrow 0 .
$$

By the Lemma 2.1, the continuity of function $k e^{*}(k, x)$ follows from that of function $k s_{11}(k)$. In proving estimate (2.58) two case may arise.

(1) The function $s_{11}(k)$ is bounded in neighborhood of point $k=0$, in which case the function $e^{*}(k, x)$ is also bounded in a neighborhood of this point, therefore, $k e^{*}(k, x) \rightarrow 0$ as $k \rightarrow 0$. Hence, the estimate (2.58) follows from the continuity of $k e^{*}(k, x)$.

(2) The function $s_{11}(k)$ is not bounded in a neighborhood of the point $k=0$, in which case there exists a sequence $k_{n} \rightarrow 0$ such that $s_{11}\left(k_{n}\right) \rightarrow \infty$. It follows from condition (2.32) that $\lim _{n \rightarrow \infty} k_{n} s_{11}\left(k_{n}\right)=O(1), \lim _{n \rightarrow \infty} \frac{s_{21}\left(k_{n}\right)}{s_{11}\left(k_{n}\right)}=-1$.

Putting $k=k_{n}$ in (2.51), passing to the limit as $k_{n} \rightarrow 0$, gives: $1+\int_{x}^{\infty} \tilde{\Phi}(x, y) \mathrm{d} y=\omega(0, x)-$ $\omega(0, x)=0$. Therefore, $\lim _{n \rightarrow \infty} k_{n} e^{*}\left(k_{n}, x\right)=0$, which proves estimate (2.58) in this case too, c.l due to the continuity of the function $k e^{*}(k, x)$.

$$
e^{*}(k, x)-e^{i k x} \in L_{2}(-\infty, \infty) .
$$

Since the function $s_{11}(k)$ satisfies the first estimate of (2.25) for large $k$, therefore it suffices to show that $e^{*}(k, x)$ is square integrable in a neighborhood of the point $k=0$. We need to show that $e^{*}(k, x)$ is a bounded function in a neighborhood of the point $k=0$. We write equality (2.52) in the form:

$$
e^{*}(k, x)=\left[s_{11}(k)+s_{21}(k)\right] \omega(-k, x)+s_{21}(k)[\omega(k, x)-\omega(-k, x)] .
$$

Taking into account that: $k s_{21}(k)=o(1)$ as $k \rightarrow 0$, we get the estimate:

$$
\begin{aligned}
& s_{21}(k)[\omega(k, x)-\omega(-k, x)]=-2 i s_{21}(k) \sin k x-2 i \int_{-\infty}^{x} K^{-}(x, \xi) s_{21}(k) \sin k \xi \mathrm{d} \xi \\
& =-2 i \frac{\sin k x}{k}\left[k s_{21}(k)\right]-2 i \int_{-\infty}^{x} K^{-}(x, \xi) \frac{\sin k \xi}{k}\left[k s_{21}(k)\right] \mathrm{d} \xi \\
& =O(x)+O\left(\int_{-\infty}^{x}|\xi|\left|K^{-}(x, \xi)\right||\mathrm{d} \xi|\right)=O(1) \text { as } k \rightarrow 0 .
\end{aligned}
$$

From this estimate and assumption (2.33), it follows that the function $e^{*}(k, x)$ is bounded in a neighborhood of the point $k=0$. Thus, the assertion $\mathbf{c}$./ is proved.

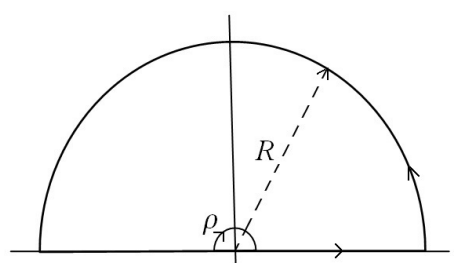

Fig. 1. The path of integration. 
Now we can prove identity (2.56). Consider a function $\left[e^{*}(k, x)-e^{i k x}\right] e^{-i k y}$ for $y<x$, which is analytic in the upper half-plane $\operatorname{Im} k>0$. Integrating this function along the contour represented in Figure 1. Due to properties a./ and $\mathbf{b} . /$ of $e^{*}(k, x)$, the integrals along the small and large semicircles tend to zero as $\rho \rightarrow 0$ and $R \rightarrow \infty$. Hence, by also the property c./ and Cauchy's Theorem: $\lim _{R \rightarrow \infty} \int_{-R}^{R}\left[e^{*}(k, x)-e^{i k x}\right] e^{-i k y} \mathrm{~d} k=0$ for $y<x$. Due to the property $\mathbf{c} . /$, there exists a function $K^{*}(x, y): \frac{1}{2 \pi} \int_{x}^{\infty}\left[e^{*}(k, x)-e^{i k x}\right] e^{-i k y} \mathrm{~d} k=K^{*}(x, y)$ for $y>x$. Hence, $K^{*}(x, y), y>x$ is the inverse Fourier transformation of the function $e^{*}(k \cdot x)-e^{i k x}$, which belongs to the space $L_{2}(-\infty, \infty)$ and

$$
e^{*}(k, x)-e^{i k x}=\int_{x}^{\infty} K^{*}(x, y) e^{i k y} \mathrm{~d} y .
$$

The Fourier transform (2.60) and its inverse Fourier transform map $L_{2}[x, \infty)$ onto $L_{2}[x, \infty)$ mutually continuously one-to-one, therefore $K^{*}(x, y) \in L_{2}(x, \infty)$. Dividing equality (2.55) by $s_{11}(k)$ :

$$
\frac{1}{s_{11}(k)} \omega(k, x)-e^{-i k x}=\int_{x}^{\infty} K^{*}(x, \xi) e^{-i k \xi} \mathrm{d} \xi-\frac{s_{21}(-k)}{s_{11}(k)}\left[e^{i k x}+\int_{x}^{\infty} K^{*}(x, \xi) e^{i k \xi} \mathrm{d} \xi\right] .
$$

The function $s_{11}(k)$ is analytical in the upper half-planeIm $k>0$ and has no zero there, then the function:

$$
\begin{aligned}
h(k, x) & =\left[\frac{1}{s_{11}(k)} \omega(k, x)-e^{-i k x}\right] e^{i k y}=\left[e^{*}(-k, x)-\frac{s_{21}(-k)}{s_{11}(k)} e^{*}(k, x)-e^{-i k x}\right] e^{i k y} \\
& =O\left(\frac{e^{-(y-x) \operatorname{Im} k}}{|k|}\right) \text { as }|k| \rightarrow \infty, y>x
\end{aligned}
$$

is also analytical in this upper half-plane.

Multiply both sides of identity (2.61) by $\frac{1}{2 \pi} e^{i k y}$, and integrate with respect to $k$, then due to the analytical property of the function $h(k, x)$ and its estimate, the left-hand side of the obtained equality vanishes when $y>x$, while the right-hand side of this equality gives:

$$
R(x+y)+K^{*}(x, y)+\int_{x}^{\infty} R(y+\xi) K^{*}(x, \xi) \mathrm{d} \xi .
$$

Therefore, the function $K^{*}(x, y)$ satisfies the integral fundamental equation (2.41). From the uniqueness of a solution of Eq. (2.41), it follows that $K^{*}(x, y)=K(x, y)$. Then by (2.43) and (2.60) $e^{*}(k, x) \equiv e(k, x)$. Since the solution of equation (2.41) is the kernel of the transformation operator, then it satisfies Eq. (2.9). Due to the uniqueness of solution of Eq. (2.9), the solution $K(x, y)$ of Eq. (2.41) is related to the potential by formula (2.48) and satisfies condition (2.49). Therefore, the restored potential (2.48) belongs to the class $\mathscr{S}$. Thus, the Schrödinger equation (2.4) is restored with the potential (2.48) satisfying condition (2.49). In addition, the negative spectrum of the restored equation is absent. Thus, the first assertion is proved.

We proceed to prove the second assertion. Let $s_{11}(k)$ and $s_{21}(k),-\infty<k<\infty$ be any given functions satisfying the sufficient conditions enumerated in Theorem 2.1. We prove that these given functions are the scattering data of the SP (2.1)-(2.2) with the restored potential belonging to the class $\mathscr{S}$. In fact, consider the SP with the restored potential (2.48) satisfying condition (2.49). Let $\tilde{s}_{11}(k)$ and $\tilde{s}_{21}(k)$ be scattering data, and let $\tilde{K}(x, y), y>x$ be the kernel of the transformation operator 
of this considered SP. Then the function $\tilde{K}(x, y)$ satisfies the equation for $y>x$ :

$$
\hat{R}(x+y)+\tilde{K}(x, y)+\int_{x}^{\infty} \hat{R}(y+\xi) \tilde{K}(x, \xi) \mathrm{d} \xi=0, \hat{R}(x+y)=-\frac{1}{2 \pi} \int_{-\infty}^{\infty} \frac{\tilde{s}_{21}(-k)}{\tilde{s}_{11}(k)} e^{i k(x+y)} \mathrm{d} k .
$$

Since the solutions $K(x, y)$ and $\tilde{K}(x, y)$ of Eqs. (2.41) and (2.62), respectively, satisfy the same integral equation (2.9) with the potential (2.48), then owing to the uniqueness of solution of Eq. (2.9), we obtain the identity: $K(x, y) \equiv \tilde{K}(x, y)$ for $y>x$. Taking this identity into account and subtracting Eq. (2.62) from Eq. (2.41), we have:

$$
R(x+y)-\hat{R}(x+y)+\int_{x}^{\infty}[R(y+\xi)-\hat{R}(y+\xi)] K(x, \xi) \mathrm{d} \xi=0 \text { for } y>x,
$$

For a sufficiently large positive $x=x_{0}$, the integral operator in homogeneous equation (2.63) is a contracting operator in the space of functions bounded on the interval $\left(x_{0}, \infty\right)$. Hence, for $x \geq x_{0}$ and $y \geq x: \hat{R}(x+y) \equiv R(x+y)$, from which it follows that, $\hat{R}(x)=R(x)$ for $x \geq 2 x_{0}$. Therefore:

$$
R(x+y)-\hat{R}(x+y)+\int_{x}^{2 x_{0}-y}[R(y+\xi)-\hat{R}(y+\xi)] K(x, \xi) \mathrm{d} \xi=0 \text { for } y>x .
$$

For fixed $x_{0}$ and $y$, Eq. (2.64) is a Volterra homogeneous, therefore

$R(x+y)-\hat{R}(x+y) \equiv 0$ for any $x$ and $y \geq x$, i.e., $\hat{R}(x)$ is identical to $R(x)$, or, in expanded form:

$$
\frac{1}{2 \pi} \int_{-\infty}^{\infty}-\frac{s_{21}(-k)}{s_{11}(k)} e^{i k x} \mathrm{~d} k=\frac{1}{2 \pi} \int_{-\infty}^{\infty}-\frac{\tilde{s}_{21}(-k)}{\tilde{s}_{11}(k)} e^{i k x} \mathrm{~d} k
$$

Then owing to uniqueness of expansion of a function in the Fourier integral: $\frac{\tilde{s}_{21}(-k)}{\tilde{s}_{11}(k)} \equiv \frac{s_{21}(-k)}{s_{11}(k)}$. Since the functions $s_{11}(k)$ and $\tilde{s}_{11}(k)$ have no zeros in the upper half-plane $\operatorname{Im} k>0$, then $s_{11}(k)$ and $\tilde{s}_{11}(k)$ are uniquely restorable from $|\tilde{R}(k)|=\left|\frac{s_{21}(-k)}{s_{11}(k)}\right|$, whence it follows that:

$$
s_{11}(k) \equiv \tilde{s}_{11}(k), s_{21}(-k) \equiv \tilde{s}_{21}(-k), s_{12}(k)=-\tilde{R}(k) s_{11}(k), \quad \tilde{r}(k)=-\frac{s_{11}(-k)}{s_{11}(k)} \tilde{R}(-k) .
$$

Thus, the ratios: $\tilde{R}(k)=-\frac{s_{12}(k)}{s_{11}(k)}$ and $\tilde{r}(k)=\frac{s_{21}(k)}{s_{11}(k)}$ satisfy conditions enumerated in Lemmas 2.2 and 2.3, respectively. Therefore, they are right- and left-reflection coefficients of the considered SP, and Eq. (2.4) is restored precisely from $\tilde{R}(k)$. Thus, the sufficiency of conditions of right-reflection and left-reflection coefficients $\tilde{R}(k)$ and $\tilde{r}(k)$ is proved. The necessity of conditions of these coefficients has been established by Lemmas 2.1, 2.2 and 2.3. The proof of Theorem 2.1 is completed.

The KdV equation (1.1) is derived from the Lax condition for compatibility of two systems:

$$
\begin{aligned}
& Y_{x}=U Y, \quad Y=\left(y_{1}, y_{2}\right), \\
& Y_{t}=V Y,
\end{aligned}
$$

where $U$ and $V$ are the given matrices having the form:

$$
U=\left(\begin{array}{cc}
0 & 1 \\
p-\lambda & 0
\end{array}\right), V=\left(\begin{array}{cc}
p_{x} & -4 \lambda-2 p \\
p_{x x}-(4 \lambda+2 p)(p-\lambda) & -p_{x}
\end{array}\right), k^{2}=\lambda
$$


The potential $p(x, t)$ for every $t>0$ belongs to the class $\mathscr{S}$, therefore the systems (2.66) and (2.67) are compatible, i.e.,

$$
y_{2 x t}=y_{2 t x} \text { or } y_{1 x x t}=y_{1 x t x} .
$$

The above equality will be referred to as the compatibility condition for systems (2.66) and (2.67). It is easy to verify that this compatibility condition is equivalent to the KdV equation (1.1). The boundary conditions (1.2) are satisfied if and only if the system (2.67) along the line $x=0$ takes the form:

$$
Y_{t}=V_{0} Y, \quad V_{0}=\left(\begin{array}{cc}
p_{x}(0, t) & -4 \lambda \\
4 \lambda^{2} & -p_{x}(0, t)
\end{array}\right), \lambda=k^{2}
$$

Upon differentiating equality (2.14) with respect to $t$, we have the equality:

$$
E_{t}(k, x, t)=W_{t}(k, x, t) s(k, t)+W(k, x, t) s_{t}(k, t),
$$

Substituting matrix expressions $E_{t}$ and $W_{t}$ into equality (2.69), using conditions (1.2), we derive the system of linear differential equations governing the time-dependence of $s(k, t)$ :

$$
s_{t}(k, t)=4 i k^{3}\left[s, \sigma_{3}\right]+p_{x}(0, t) \sigma_{1} s(k, t), \quad \sigma_{1}=\left(\begin{array}{ll}
0 & 1 \\
1 & 0
\end{array}\right), \sigma_{3}=\left(\begin{array}{cc}
1 & 0 \\
0 & -1
\end{array}\right) .
$$

The time-dependence of the scattering matrix $s(k, t)$ defined by system (2.70) implicitly depends on the time $t$. This is the main difference between the IBVP (1.1)-(1.2)-(1.3) and the Cauchy problem, therein lies the difficulty in passing from the Cauchy problem to this IBVP. The system (2.70) is undetermined, because the function $p_{x}(0, t)$ entering coefficients of this system is unknown. In Sec. 3 we shall prove that the unknown object $p_{x}(0, t)$ can be expressed through entries of the given $s(k)$.

\section{The self-conjugate problem}

The system (2.68) describes the time-evolution of the eigenfunction for the boundary point $x=0$. Using the linear change of dependent variables: $Y(k, t)=J(k) \tilde{y}(\mu, t)$, we reduce (2.68) to the form:

$$
\tilde{y}_{t}(k, t)=\left(J^{-1} V J\right) \tilde{y}(k, t),
$$

where $J$ is a matrix, which is to be taken so that $J^{-1} \sigma_{3} J=\sigma_{1}$. From the equality: $J \sigma_{1}=\sigma_{3} J$, it follows that the matrix $J$ takes the form: $J=\left(\begin{array}{cc}\alpha & \alpha \\ -\beta & \beta\end{array}\right), \alpha$ and $\beta$ are arbitrary constants and $\alpha \neq 0$, $\beta \neq 0$. Taking $\alpha=1$ and $\beta= \pm i k$, which are the roots of the equation: $\beta+k^{2}=0$, we reduce system (3.1) to the form:

$$
\tilde{y}_{t}(\mu, t)=\left( \pm 4 i \mu \sigma_{3}+p_{x}(0, t) \sigma_{1}\right) \tilde{y}(\mu, t), \mu=k^{3} .
$$

Let $\beta=-i k$, then the matrix $J$ in (3.1) coincides with the matrix $T(k)$ defined by:

$$
J(k)=\left(\begin{array}{cc}
1 & 1 \\
i k & -i k
\end{array}\right)=T(k), \quad Y(k, t)=T(k) \tilde{y}(\mu, t), \mu=k^{3}, \quad 0 \leq t<\infty .
$$


Thus, by virtue of the linear change of dependent variables given by (3.3), we lead system (3.2) into the system of first-order ordinary differential equations on the half-line:

$$
i \sigma_{3} \tilde{y}_{t}(\mu, t)+C(t) \tilde{y}(\mu, t)=4 \mu \tilde{y}(\mu, t), \mu=k^{3}, 0 \leq t<\infty,
$$

where the potential matrix $C(t)$ is self-conjugate:

$$
C(t)=\left(\begin{array}{cc}
0 & c_{1}(t) \\
c_{2}(t) & 0
\end{array}\right)=\overline{C(t)}
$$

$c_{1}(t)=-i p_{x}(0, t), c_{2}(t)=i p_{x}(0, t)$, the potential $p_{x}(0, t)$ is a real-valued function.

Denote $\tilde{y}(\mu, t)$ by $y(\mu, t)$ and consider the self-conjugate SP generated by system (3.4):

$$
i \sigma_{3} y_{t}(\mu, t)+C(t) y(\mu, t)=4 \mu y(\mu, t), \mu=k^{3}, 0 \leq t<\infty
$$

with the boundary condition at the origin $t=0$ :

$$
y_{1}(\mu, 0)=y_{2}(\mu, 0) .
$$

We also consider the problem for system (3.6) for real $\mu$ with boundary conditions at infinity:

$$
y_{1}(\mu, t)=A(\mu) e^{-4 i \mu t}+o(1), \quad y_{2}(\mu, t)=B(\mu) e^{4 i \mu t}+o(1), \quad(t \rightarrow \infty) .
$$

Assume that the potential function $p_{x}(0, t)$ in (3.5) satisfies the estimate:

$$
\left|p_{x}(0, t)\right| \leq \frac{\text { Const. }}{1+t^{1+\varepsilon}}, \quad t>0, \varepsilon>0 .
$$

The problems (3.6), (3.8) and (3.6)-(3.7) with the potential satisfying estimate (3.9) have been solved in [14]. Since the potential matrix (3.5) is a particular case of the potential self-conjugate matrix of the problem investigated in [14], then the following Propositions are deduced from corresponding assertions proved in [14] without proving.

Proposition 3.1. The problem (3.6)-(3.7) has a unique bounded solution $\left(y_{1}(\mu, t), y_{2}(\mu, t)\right)$ for real $\mu$ such that for any given number $A(\mu)$ there exists a unique number $B(\mu)$ defined from $A(\mu)$ so that the asymptotics (3.8) are satisfied. This solution has the representation:

$$
\begin{aligned}
& y_{1}(\mu, t)=A(\mu) e^{-4 i \mu t}\left(1+\int_{0}^{\infty} H_{11}(t, t+\xi) e^{-4 i \mu \xi} \mathrm{d} \xi\right)+B(\mu) e^{4 i \mu t} \int_{0}^{\infty} H_{21}(t, t+\xi) e^{4 i \mu \xi} \mathrm{d} \xi \\
& y_{2}(\mu, t)=A(\mu) e^{-4 i \mu t} \int_{0}^{\infty} H_{21}(t, t+\xi) e^{-4 i \mu \xi} \mathrm{d} \xi+B(\mu) e^{4 i \mu t}\left(1+\int_{0}^{\infty} H_{11}(t, t+\xi) e^{4 i \mu \xi}\right),
\end{aligned}
$$

where the matrix $H(t, s)=\left(\begin{array}{l}H_{11}(t, s) H_{21}(t, s) \\ H_{21}(t, s) H_{11}(t, s)\end{array}\right), 0 \leq t \leq s$ is an analog of kernel of the transformation operator. The functions $H_{11}(t, s)$ and $H_{21}(t, s)$ satisfy the estimate:

$$
\left|H_{j 1}(t, s)\right| \leq \frac{\text { Const. }}{1+(t+s)^{1+\varepsilon}}, \quad 0 \leq t \leq s, \quad j=1,2 .
$$

Using condition (3.5), wherein the functions $c_{1}(t), c_{2}(t)$ are pure imaginary functions, from the integral equations for kernels we obtain that the solutions $H_{j k}(t, s), j, k=1,2$ of these equations 
P.L. Vu / The description of reflection coefficients of the scattering problems ...

are real-valued functions, and

$$
H_{11}(t, s)=H_{22}(t, s), H_{21}(t, s)=H_{12}(t, s), 0 \leq t \leq s
$$

The kernel function $H_{21}(t, t+\xi)$ is related to the potential $p_{x}(0, t)$ by the formula:

$$
-2 H_{21}(t, t)=p_{x}(0, t) \text { for } t>0 .
$$

Proposition 3.2. There exist the bounded Jost solutions $e^{(1)}(\mu, t)=\left(e_{1}^{(1)}(\mu, t), e_{2}^{(1)}(\mu, t)\right)$ and $e^{(2)}(\mu, t)=\left(e_{1}^{(2)}(\mu, t), e_{2}^{(2)}(\mu, t)\right)$ of system problem (3.6) with the boundary conditions at infinity:

$$
\begin{gathered}
e^{(1)}(\mu, t) e^{4 i \mu t}=(1+o(1), o(1)), \quad \operatorname{Im} \mu \leq 0 \quad(t \rightarrow \infty), \\
e^{(2)}(\mu, t) e^{-4 i \mu t}=(o(1), 1+o(1)), \quad \operatorname{Im} \mu \geq 0 \quad(t \rightarrow \infty) .
\end{gathered}
$$

By an argument analogous to problem (3.6)-(3.7) on the half-line $0 \leq t<\infty$, for any $t \geq 0$ we consider the problem generated by system (3.6) on a half-line $t \leq \tau<\infty$ with the boundary condition at $\tau=t$ :

$$
z_{1}(\mu, t, t)=z_{2}(\mu, t, t)
$$

and the problem for this system with the boundary condition at infinity:

$$
z_{1}(\mu, \tau, t)=A(\mu, t) e^{-4 i \mu(\tau-t)}+o(1), z_{2}(\mu, \tau, t)=B(\mu, t) e^{4 i \mu(\tau-t)}+o(1), \quad(\tau \rightarrow \infty), \quad t \leq \tau<\infty .
$$

Definition 3.1. The one-to-one correspondence between numbers $A(\mu, t)$ and $B(\mu, t)$ determines the scattering function $S(\mu, t): S(\mu, t) A(\mu, t)=B(\mu, t),-\infty<\mu<\infty$ for the SP generated by system (3.6) on the half-line: $t \leq \tau<\infty$ with condition (3.13).

By Definition 3.1 and using (3.10), (3.13), we derive the factorization of $S(\mu, t)$ :

$$
S(\mu, t)=\left(1+H_{-}(\mu, t)\right)\left(1+H_{+}(\mu, t)\right)^{-1},-\infty<\mu<\infty,
$$

where $H_{-}(\mu, t)=\int_{0}^{\infty} H(t, \xi) e^{-4 i \mu \xi} \mathrm{d} \xi, H_{+}(\mu, t)=\int_{0}^{\infty} H(t, \xi) e^{4 i \mu \xi} \mathrm{d} \xi, H(t, \xi)=H_{11}(t, t+\xi)-$ $H_{21}(t, t+\xi)$ and $H_{j 1}(t, t+\xi), j=1,2$ satisfies estimate (3.11).

By virtue of estimate (3.11) and the self-conjugate property of matrix (3.5), the function $H(t, \xi)$ is absolutely integrable with respect to $\xi$, the numerator $1+H_{-}(\mu, t)$ and denominator $1+H_{+}(\mu, t)$ of ratio (3.14) are different from zero and analytic in the half-planes $\operatorname{Im} \mu \leq 0$ andim $\mu \geq 0$, respectively. There exists an absolutely integrable with respect to $\xi$ function $K(t, \xi)$ such that

$$
\left(1+\int_{0}^{\infty} H(t, \xi) e^{4 i \mu \xi} \mathrm{d} \xi\right)^{-1}=1+\int_{0}^{\infty} K(t, \xi) e^{4 i \mu \xi} \mathrm{d} \xi
$$

Furthermore, for any $t \geq 0$ the scattering function $S(\mu, t)$ possesses the property:

$$
\overline{S(\mu, t)}=S^{-1}(\mu, t)=S(-\mu, t), \quad-\infty<\mu<\infty .
$$


P.L. Vu / The description of reflection coefficients of the scattering problems ...

Proposition 3.3. For any $t \geq 0$ the scattering functions $S(\mu, t)-1$ and $S^{-1}(\mu, t)-1$ for the $S P$ (3.6), (3.13) are the Fourier transformations:

$$
\begin{aligned}
& S(\mu, t)=1+F(\mu, t)=1+\int_{-\infty}^{\infty} f(t, \xi) e^{4 i \mu \xi} \mathrm{d} \xi \\
& S^{-1}(\mu, t)=1+G(\mu, t)=1+\int_{-\infty}^{\infty} g(t, \xi) e^{4 i \mu \xi} \mathrm{d} \xi
\end{aligned}
$$

where $f(t, \xi)$ and $g(t, \xi)$ are real-valued functions defined by the formulas:

$$
\begin{aligned}
& f(t, \xi)=H(t,-\xi)+K(t, \xi)+\int_{0}^{\infty} K(t, \zeta) H(t, \zeta-\xi) \mathrm{d} \zeta \\
& g(t, \xi)=H(t, \xi)+K(t,-\xi)+\int_{-\infty}^{0} K(t,-\zeta) H(t, \xi-\zeta) \mathrm{d} \zeta .
\end{aligned}
$$

Proposition 3.4. There exists uniquely a solution $\varphi(\mu, t)=\left(\varphi_{1}(\mu, t), \varphi_{2}(\mu, t)\right)$ of (3.6) with the initial condition: $\varphi_{1}(\mu, 0)=\varphi_{2}(\mu, 0)=1$. The function $\varphi(\mu, t)$ is entire analytic of $\mu$, and:

$$
\begin{aligned}
& D^{-1}(\mu) \varphi(\mu, t)=e^{(1)}(\mu, t)+S(\mu) e^{(2)}(\mu, t), \quad \operatorname{Im} \mu \geq 0, \\
& N^{-1}(\mu) \varphi(\mu, t)=S^{-1}(\mu) e^{(1)}(\mu, t)+e^{(2)}(\mu, t), \quad \operatorname{Im} \mu \leq 0,
\end{aligned}
$$

where $N(\mu)=e_{1}^{(1)}(\mu, 0)-e_{2}^{(1)}(\mu, 0)=1+H_{-}(\mu, 0) \neq 0$ for $\operatorname{Im} \mu \leq 0$,

$$
\begin{aligned}
& D(\mu)=e_{2}^{(2)}(\mu, 0)-e_{1}^{(2)}(\mu, 0)=\overline{N(\mu)} \neq 0 \text { for } \operatorname{Im} \mu \geq 0, \\
& N(\mu)=1+o(1) \quad(|\mu| \rightarrow \infty), D(\mu)=1+o(1) \quad(|\mu| \rightarrow \infty), \\
& S(\mu)=\frac{N(\mu)}{D(\mu)}, S^{-1}(\mu)=\overline{S(\mu)}=S(-\mu), \quad-\infty<\mu<\infty,
\end{aligned}
$$

Proposition 3.5. For any $t \geq 0$ the functions $f(t, \xi)$ and $g(t, \xi)$ defined by (3.18) and (3.19) are closely related to $f(\xi)$ and $g(\xi)$, respectively by the formula:

$$
\begin{aligned}
& f(t, \xi)=f(\xi-2 t) \text { for } \xi<0, g(t, \xi)=g(\xi+2 t) \text { for } \xi>0, \\
& f(-\xi-2 t)=g(\xi+2 t) \text { for } \xi>0 .
\end{aligned}
$$

The functions $f(-\xi)$ and $g(\xi), \xi>0$ satisfy the estimate of the type (3.9):

$$
|f(-\xi)| \leq \frac{\text { const }}{1+\xi^{1+\varepsilon}}, \quad|g(\xi)| \leq \frac{\text { const }}{1+\xi^{1+\varepsilon}}, \quad \xi>0, \quad \varepsilon>0 .
$$

Proposition 3.6. For every fixed $t \geq 0$ the following Fredholm system:

$$
\left\{\begin{array}{l}
H_{22}(t, t+\xi)+\int_{0}^{\infty} H_{21}(t, t+\zeta) g(2 t+\xi+\zeta) \mathrm{d} \zeta=0 \\
g(2 t+\xi)+H_{21}(t, t+\xi)+\int_{0}^{\infty} H_{22}(t, t+\zeta) g(2 t+\xi+\zeta) \mathrm{d} \zeta=0
\end{array}\right.
$$

has a unique solution $H_{22}(t, t+\xi), H_{21}(t, t+\xi)$ in the space $L_{1}[0, \infty)$.

Proposition 3.7. For the given function $S(\mu)$ to be the scattering function for the self-conjugate problem (3.6)-(3.7), it is necessary and sufficient that there exists a function $S(\mu, t)$ such that $S(\mu)=$ $S(\mu, 0)$ and

1. the function $S(\mu, t)$ admits the factorization of the form (3.14); 
2. for any $t \geq 0$ the functions $S(\mu, t)-1$ and $S^{-1}(\mu, t)-1$ are the Fourier transformations (3.16) and (3.17) of the functions (3.18) and (3.19), respectively;

3. for any $t \geq 0$ the functions $f(t, \xi)$ and $g(t, \xi)$ are closely related to $f(-\xi)$ and $g(\xi)$ by formula (3.22), respectively. $f(-\xi)$ and $g(\xi)$ are real-valued functions satisfying the estimate of type (3.9).

To solve the inverse SP (3.6)-(3.7) for finding the unknown object $p_{x}(0, t)$, we need to find the unknown scattering function $S(\mu)$ of this problem first. To find $S(\mu)$, we express the function $S(\mu)$ through known elements of the scattering matrix $s(k)$ of the SP (2.1)-(2.2). This is the key step to recover the potential matrix (3.5), i.e., the system (3.6).

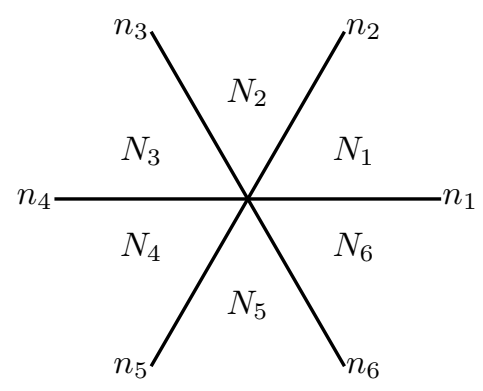

Fig. 2. The conjugation contour.

The matter is that the first SP (2.1), (2.2) and the second SP (3.6)-(3.7) are formulated on two different spectral planes. The first SP is considered on the $k$-plane, whereas the second SP is considered on the $\mu$-plane, $\left(\mu=k^{3}\right)$. To compare functions on the $k$-plane with those on the $\mu$ plane, we use the conjugation contour. The contour $\operatorname{Im} \mu=\operatorname{Im} k^{3}=0$ splits into a system of rays $\left\{n_{j}\right\}_{j=1}^{6}$ coming from the origin of the $k$-plane with the slope angles $\gamma_{j}=(j-1) \pi / 3$ with respect to the positive direction of the line $\operatorname{Im} k=0$. We denote by $N_{j}$ the interior sector confined between the rays $n_{j+1}$ and $n_{j}$ (see the Figure 2). Taking into account that $\arg \mu=\arg k^{3}=3 \arg k$, we have:

$$
\begin{aligned}
& \text { If } k \in N_{1} \cup N_{3} \cup N_{5} \text {, then } \operatorname{Im} \mu>0 . \\
& \text { If } k \in N_{2} \cup N_{4} \cup N_{6} \text {, then } \operatorname{Im} \mu<0 .
\end{aligned}
$$

We compose the matrix functions $\psi^{+}(\mu, t)$ and $\psi^{-}(\mu, t)$ according to the rule:

$$
\begin{aligned}
& \psi^{+}(\mu, t)=\left(\frac{\varphi(\mu, t)}{D(\mu)}, e^{(2)}(\mu, t)\right) e^{4 i \mu t \sigma_{3}} \text { for } k \in N_{1} \cup N_{3} \cup N_{5}, \\
& \psi^{-}(\mu, t)=\left(e^{(1)}(\mu, t), \frac{\varphi(\mu, t)}{N(\mu)}\right) e^{4 i \mu t \sigma_{3}} \text { for } k \in N_{2} \cup N_{4} \cup N_{6} .
\end{aligned}
$$

Since the matrix functions $\left(\frac{\varphi(\mu, t)}{D(\mu)}, e^{(2)}(\mu, t)\right)$ and $\left(e^{(1)}(\mu, t), \frac{\varphi(\mu, t)}{N(\mu)}\right)$ satisfy system (3.6), then the matrix functions $\psi^{+}(\mu, t)$ and $\psi^{-}(\mu, t)$ satisfy system (2.70). Hence, all the matrix functions $s(k, t)$, $\psi^{+}(\mu, t)$ and $\psi^{-}(\mu, t)$ satisfy the same system (2.70). It is easily persuaded that the functions $\psi^{+}(\mu, t)$ and $\psi^{-}(\mu, t)$ are the fundamental solutions of system (2.70). Denote the columns of $s(k, t)$ 
by $s_{1}(k, t)=\left(s_{11}(k, t), s_{21}(k, t)\right), s_{2}(k, t)=\left(s_{12}(k, t), s_{22}(k, t)\right)$. Both columns are solutions of system (2.70), but they are defined in different half-planes, therefore we consider them separately for convenience. Every column solution $s_{j}(k, t), j=1,2$ can be represented in a form:

$$
\begin{aligned}
& s_{1}(k, t)=\psi^{+}(\mu, t)\left(\begin{array}{l}
\alpha(k, t) \\
\beta(k, t)
\end{array}\right) \text { for } k \in N_{1} \cup N_{3}, \\
& s_{1}(k, t)=\psi^{-}(\mu, t)\left(\begin{array}{l}
\alpha(k, t) \\
\beta(k, t)
\end{array}\right) \text { for } k \in N_{2}, \\
& s_{2}(k, t)=\psi^{-}(\mu, t)\left(\begin{array}{l}
\alpha(k, t) \\
\beta(k, t)
\end{array}\right) \text { for } k \in N_{4} \cup N_{6}, \\
& s_{2}(k, t)=\psi^{+}(\mu, t)\left(\begin{array}{l}
\alpha(k, t) \\
\beta(k, t)
\end{array}\right) \text { for } k \in N_{5},
\end{aligned}
$$

where the coefficients $\alpha(k, t)$ and $\beta(k, t)$ are unknown, which will be defined below.

The functions $s_{1}(k, t)$ and $s_{2}(k, t)$ are defined in the half-planes $\operatorname{Im} k \geq 0$ and $\operatorname{Im} k \leq 0$, respectively. We compose the matrix functions $\Phi^{+}$and $\Phi^{-}$by columns of matrices (2.5) and (2.6) at $x=0$ according to the rule:

$$
\begin{aligned}
& \Phi^{+}(k, 0, t)=\left(s_{11}^{-1}(k, t) E^{+}(k, 0, t), W^{+}(k, 0, t)\right) \operatorname{Im} k>0, \\
& \Phi^{-}(k, 0, t)=\left(W^{-}(k, 0, t), s_{22}^{-1}(k, t) E^{-}(k, 0, t)\right) \operatorname{Im} k<0 .
\end{aligned}
$$

Using the consistency condition for systems (2.66) and (2.67) in the quarter of the plane $x \geq 0$, $t \geq 0$, we calculate $\alpha(k, 0)$ and $\beta(k, 0)$. In view of system (2.68) for the eigenfunction along the line $x=0$ and the change of variables (3.3), this consistency condition means that the matrix functions $T(k) \psi^{+}(\mu, t), T(k) \psi^{-}(\mu, t)$ defined on $\mu$-plane and $\Phi^{+}(k, 0, t), \Phi^{-}(k, 0, t)$ defined on $k$-plane must be consistent at the corner point $(x, t)=(0,0)$ for all values of $k$. Making use of this fact, we calculate $\alpha(k, 0)$ and $\beta(k, 0)$ for $k \in N_{j}, j=1,2, \ldots, 6$, [11]:

$$
\begin{aligned}
& \alpha(k, 0)=s_{11}(k, 0)-r_{12}\left(\omega^{2} k\right) s_{21}(k, 0), \beta(k, 0)=s_{21}(k, 0) \text { for } k \in N_{1}, \\
& \alpha(k, 0)=s_{11}(k, 0), \beta(k, 0)=0 \text { for } k \in N_{2}, \\
& \alpha(k, 0)=s_{11}(k, 0)-r_{12}(\omega k) s_{21}(k, 0), \beta(k, 0)=s_{21}(k, 0) \text { for } k \in N_{3}, \\
& \alpha(k, 0)=s_{12}(k, 0), \beta(k, 0)=s_{22}(k, 0)-r_{21}\left(\omega^{2} k\right) s_{12}(k, 0) \text { for } k \in N_{4}, \\
& \alpha(k, 0)=0, \beta(k, 0)=s_{22}(k, 0) \text { for } k \in N_{5}, \\
& \alpha(k, 0)=s_{12}(k, 0), \beta(k, 0)=s_{22}(k, 0)-r_{21}(\omega k) s_{12}(k, 0) \text { for } k \in N_{6} .
\end{aligned}
$$

Using formula (3.21), (3.26) and (3.27), from (3.28)-(3.31) for $t=0$, we get:

$$
\begin{aligned}
& D(\mu) \beta(k, 0)= \begin{cases}s_{21}(k, 0)-s_{11}(k, 0) & \text { for } k \in N_{1} \cup N_{3} \\
s_{22}(k, 0)-s_{12}(k, 0) & \text { for } k \in N_{5}\end{cases} \\
& N(\mu) \alpha(k, 0)= \begin{cases}s_{12}(k, 0)-s_{22}(k, 0) & \text { for } k \in N_{4} \cup N_{6} \\
s_{11}(k, 0)-s_{21}(k, 0) & \text { for } k \in N_{2} .\end{cases}
\end{aligned}
$$

Here the coefficients $\alpha(k, 0), \beta(k, 0)$ are calculated by formulas (3.32)-(3.37):

$$
\begin{aligned}
& \beta(k, 0)=s_{21}(k, 0) \text { for } k \in N_{1} \cup N_{3}, \alpha(k, 0)=s_{12}(k, 0) \text { for } k \in N_{4} \cup N_{6}, \\
& \beta(k, 0)=s_{22}(k, 0) \text { for } k \in N_{5}, \alpha(k, 0)=s_{11}(k, 0) \text { for } k \in N_{2} .
\end{aligned}
$$


Due to (2.20), from (3.40) we have the asymptotics as $|k| \rightarrow \infty$ :

$$
\beta(k, 0)=s_{21}(k, 0) \rightarrow 0 \text { for } k \in N_{1} \cup N_{3}, \quad \alpha(k, 0)=s_{12}(k, 0) \rightarrow 0 \text { for } k \in N_{4} \cup N_{6},
$$

but the equalities (3.38) for $k \in N_{1} \cup N_{3}$ and (3.39) for $k \in N_{4} \cup N_{6}$ do not tend to zero when $|k| \rightarrow \infty$. Thus, the sought coefficients $\beta(k, 0)$ and $\alpha(k, 0)$ in (3.38) and (3.39) are determined by formulas (3.41). The equalities (3.38) for all $k \in N_{5}$ and (3.39) for all $k \in N_{2}$ are derived for establishing relationship between the sought quantities $N(\mu), D(\mu)$ and entries $s_{i j}(k)$ of the known scattering matrix $s(k)$. From these derived equalities, using Lemma 2.1, we obtain the formulas for calculating the sought quantities expressed through given entries of $s(k)$ :

$$
\begin{aligned}
& D(\mu)=1-\frac{s_{12}(k, 0)}{s_{22}(k, 0)} \text { for } k \in N_{5}, \operatorname{Im} \mu>0, \\
& N(\mu)=\overline{D(\mu)}=1-\frac{s_{21}(k, 0)}{s_{11}(k, 0)} \text { for } k \in N_{2}, \operatorname{Im} \mu<0 .
\end{aligned}
$$

Using the self-conjugate property of the SP (3.6)-(3.7) and (2.16), (2.19), (2.20) we get:

$$
\begin{aligned}
& D(\mu)=1-\frac{i k e(-k)+e_{x}(-k)}{i k e(-k)-e_{x}(-k)}=2+o(1) \text { as } k \rightarrow 0, D(\mu)=1+o(1) \text { as }|\mu| \rightarrow \infty, \\
& N(\mu)=1-\frac{i k e(k)-e_{x}(k)}{i k e(k)+e_{x}(k)}=2+o(1) \text { as } k \rightarrow 0, N(\mu)=1+o(1) \text { as }|\mu| \rightarrow \infty, \\
& S(\mu)=\frac{N(\mu)}{D(\mu)}, \overline{S(\mu)}=S^{-1}(\mu)=S(-\mu)-\infty<\mu<\infty .
\end{aligned}
$$

Hence, the found quantities $D(\mu), N(\mu)$ and $S(\mu)$ satisfy all properties assembled in Proposition 3.4.

\section{The time-evolution of $s(k, t)$ and solution of the IBVP}

The differential equations for functions $s_{1}(k, t)$ and $s_{2}(k, t)$ are derived from system (2.70) for columns of the matrix $s(k, t)$ :

$$
\begin{aligned}
& \frac{\mathrm{d}}{\mathrm{d} t}\left(\begin{array}{l}
s_{11}(k, t) \\
s_{21}(k, t)
\end{array}\right)=8 i k^{3}\left(\begin{array}{c}
0 \\
s_{21}(k, t)
\end{array}\right)+p_{x}(0, t)\left(\begin{array}{c}
s_{21}(k, t) \\
s_{11}(k, t)
\end{array}\right), \quad \operatorname{Im} k \geq 0, \\
& \frac{\mathrm{d}}{\mathrm{d} t}\left(\begin{array}{c}
s_{12}(k, t) \\
s_{22}(k, t)
\end{array}\right)=-8 i k^{3}\left(\begin{array}{c}
s_{12}(k, t) \\
0
\end{array}\right)+p_{x}(0, t)\left(\begin{array}{c}
s_{22}(k, t) \\
s_{12}(k, t)
\end{array}\right), \quad \operatorname{Im} k \leq 0 .
\end{aligned}
$$

Further, differentiating equality (3.28) with respect to $t$ and taking into account that the matrix functions $s(k, t)$ and $\psi^{+}(\mu, t)=\left[\psi_{i j}^{+}(\mu, t)\right]$ satisfy the same system (2.70), gives:

$$
\begin{aligned}
\frac{\mathrm{d}}{\mathrm{d} t}\left(\begin{array}{c}
s_{11}(k, t) \\
s_{21}(k, t)
\end{array}\right)= & \left\{8 i k^{3}\left(\begin{array}{cc}
0 & -\psi_{12}^{+}(\mu, t) \\
\psi_{21}^{+}(\mu, t) & 0
\end{array}\right)+p_{x}(0, t) \sigma_{1} \psi^{+}(\mu, t)\right\} \times \\
& \left(\begin{array}{l}
\alpha(k, t) \\
\beta(k, t)
\end{array}\right)+\psi^{+}(\mu, t) \frac{\mathrm{d}}{\mathrm{d} t}\left(\begin{array}{c}
\alpha(k, t) \\
\beta(k, t)
\end{array}\right), k \in N_{1} .
\end{aligned}
$$


By comparison of the last equality with equality (4.1), using (3.28) for $k \in N_{1}$ we derive the evolution equation:

$$
\begin{aligned}
& \frac{\mathrm{d}}{\mathrm{d} t}\left(\begin{array}{c}
\alpha(k, t) \\
\beta(k, t)
\end{array}\right)=8 i k^{3} \beta(k, t)\left(\psi^{+}(\mu, t)\right)^{-1}\left(\begin{array}{l}
\psi_{12}^{+}(\mu, t) \\
\psi_{22}^{+}(\mu, t)
\end{array}\right)= \\
& 8 i k^{3} \beta(k, t)\left(\psi^{+}(\mu, t)^{-1}\left(\psi^{+}(\mu, t)\right)\left(\begin{array}{l}
0 \\
1
\end{array}\right)=8 i k^{3} \beta(k, t)\left(\begin{array}{l}
0 \\
1
\end{array}\right), k \in N_{1} .\right.
\end{aligned}
$$

Using (3.32), from (4.3) we obtain the explicit formulas for the coefficient $(\alpha(k, t), \beta(k, t))$ :

$$
\alpha(k, t)=\alpha(k, 0)=s_{11}(k, 0)-r_{12}\left(\omega^{2} k\right) s_{21}(k, 0), \beta(k, t)=s_{21}(k, 0) e^{8 i k^{3} t} \text { for } k \in N_{1} .
$$

Thus, the evolution in time $t, t>0$ of $s_{1}(k, t)$ for $k \in N_{1}$ is derived from (3.28) with this coefficient:

$$
s_{1}(k, t)=\psi^{+}(\mu, t)\left(\begin{array}{c}
s_{11}(k, 0)-r_{12}\left(\omega^{2} k\right) s_{21}(k, 0) \\
s_{21}(k, 0) e^{8 i k^{3} t}
\end{array}\right) \text { for } k \in N_{1} .
$$

In the same way as in the previous case, by using (4.1), (4.2), (3.28)-(3.31) and (3.33)-(3.37), analogously derive:

$$
\begin{aligned}
& s_{1}(k, t)=\psi^{-}(\mu, t)\left(\begin{array}{c}
s_{11}(k, 0) \\
0
\end{array}\right) \text { for } k \in N_{2}, \\
& s_{1}(k, t)=\psi^{+}(\mu, t)\left(\begin{array}{c}
s_{11}(k, 0)-r_{12}(\omega k) s_{21}(k, 0) \\
s_{21}(k, 0) e^{8 i k^{3} t}
\end{array}\right) \text { for } k \in N_{3} . \\
& s_{2}(k, t)=\psi^{-}(\mu, t)\left(\begin{array}{c}
s_{12}(k, 0) e^{-8 i k^{3} t} \\
s_{22}(k, 0)-r_{21}\left(\omega^{2} k\right) s_{12}(k, 0)
\end{array}\right) \text { for } k \in N_{4}, \\
& s_{2}(k, t)=\psi^{+}(\mu, t)\left(\begin{array}{c}
0 \\
s_{22}(k, 0)
\end{array}\right) \text { for } k \in N_{5}, \\
& s_{2}(k, t)=\psi^{-}(\mu, t)\left(\begin{array}{c}
s_{12}(k, 0) e^{-8 i k^{3} t} \\
s_{22}(k, 0)-r_{21}(\omega k) s_{12}(k, 0)
\end{array}\right) \text { for } k \in N_{6}
\end{aligned}
$$

Hence, the obtained columns $s_{1}(k, t)$ and $s_{2}(k, t)$ are expressible by expansion formulas (4.4)-(4.6) and (4.7)-(4.9), respectively in their sectors in terms of entries of $s(k, 0)$ and fundamental solutions $\psi^{ \pm}(\mu, t)$ of system (2.70). The solutions $\Psi^{ \pm}(\mu, t)$ are calculated from known conditions (1.2) and (1.3). The condition $t \geq 0$ is important precisely here. Indeed, for $t<0$ the functions $s_{1}(k, t)$ and $s_{2}(k, t)$ are therefore, no longer bounded at infinity of $t$.

We are now to solve the IBVP (1.1)-(1.3). By Theorems 2.1 and results presented in Secs. 3 and 4 , this problem is reduced to that of solving the GLM time-dependent equation (2.41):

$$
R(x+y, t)+K(x, y, t)+\int_{x}^{\infty} R(y+\xi, t) K(x, \xi, t) \mathrm{d} \xi=0 \text { for } y>x, t \geq 0,
$$

where $x$ and $t$ enter Eq. (4.10) as parameters, $K(x, y, t), y>x$ is an unknown function of $y$ for every $(x, t) \in[0, \infty) \times[0, \infty)$, and $R(x+y, t)$ is the function defined by (2.40) for $y>x, t>0$ :

$$
R(x+y, t)=\frac{1}{2 \pi} \int_{-\infty}^{\infty} e^{i k(x+y)} \tilde{R}(k, t) \mathrm{d} k, \quad \tilde{R}(k, t)=-\frac{s_{12}(k, t)}{s_{11}(k, t)}, s_{12}(k, t)=s_{21}(-k, t),
$$

and for every $t>0$ the functions $s_{11}(k, t)$ and $s_{21}(k, t)$ satisfy the sufficient conditions of Theorem 2.1 . 
From the unique solvability of Eq. (2.41), it follows that for every $t \geq 0$ the Eq. (4.10) has a unique solution in either $L_{2}[x, \infty)$ or $L_{1}[x, \infty)$. By condition (2.38) and Parseval's relation, we find that $\|R\|_{L_{2}}<1$ in $L_{2}[x, \infty)$. Consequently, Eq. (4.10) can be solved by the method of successive approximations. The solution of Eq. (4.10) may be represented as a convergent Neumann series:

$$
\begin{aligned}
K(x, y ; t)= & -R(x+y, t)+\int_{x}^{\infty} R\left(y+\xi_{1} ; t\right) R\left(x+\xi_{1}, t\right) \mathrm{d} \xi_{1} \\
& -\int_{x}^{\infty} R\left(\xi_{2}+y, t\right) \mathrm{d} \xi_{2} \int_{x}^{\infty} R\left(\xi_{1}+\xi_{2}, t\right) R\left(x+\xi_{1}, t\right) \mathrm{d} \xi_{1}+\cdots
\end{aligned}
$$

The solution $p(x, t)$ of the IBVP (1.1)-(1.3) is constructed by formula (2.11) expressed through the solution (4.12) of Eq. (4.10):

$$
p(x, t)=-2 \frac{\mathrm{d}}{\mathrm{d} x} K(x, x ; t) .
$$

Hence, the solution (4.13) of Eq. (1.1) corresponding to solution (4.12) of Eq. (4.10) is determined by:

$$
\begin{aligned}
& p(x, t)=2 \frac{\mathrm{d}}{\mathrm{d} x} R(2 x, t)+2 R^{2}(2 x, t)-4\left\{\int_{x}^{\infty} R(x+\xi, t) R_{x}(x+\xi, t) \mathrm{d} \xi+\right. \\
& \left.R(2 x, t) \int_{x}^{\infty} R^{2}(x+\xi, t) \mathrm{d} \xi-\int_{x}^{\infty} R_{x}\left(x+\xi_{2}, t\right) \mathrm{d} \xi_{2} \int_{x}^{\infty} R\left(\xi_{1}+\xi_{2}, t\right) R\left(x+\xi_{1}, t\right) \mathrm{d} \xi_{1}\right\}+\cdots
\end{aligned}
$$

One can verify directly that the solution (4.14) satisfies Eq. (1.1) to any desired order in powers of $R$. The presentation (4.14) in formality is similar to that of the solution of the KdV equation with the positive coefficient of the dispersive term on the whole-line, that evolves from a purely continuous spectrum, [9].

By Theorem 2.1 the solution of Eq. (4.10) coincides with the kernel of the transformation operator of the SP (2.1)-(2.2) with potential (4.14). Hence, $p(x, t)$ determined by (4.14) belongs to the class $\mathscr{S}$, and therefore it satisfies condition (2.49).

Consider the asymptotic behaviour of $s_{1}(k, t)$ and $s_{2}(k, t)$ at infinity of time $(t)$. Substituting (3.26) into (4.4), (4.6) and (4.8), using Propositions 3.2, and 3.4 gives:

$$
\begin{aligned}
& \left(\begin{array}{l}
s_{11}(k, t) \\
s_{21}(k, t)
\end{array}\right)=\left(\begin{array}{c}
s_{11}(k, 0)-r_{12} s_{21}(k, 0)+o(1) \\
o(1)
\end{array}\right) \text { as } t \rightarrow \infty \text { for } k \in N_{1} \cup N_{3}, \operatorname{Im} k^{3}>0, \\
& \left(\begin{array}{c}
s_{12}(k, t) \\
s_{22}(k, t)
\end{array}\right)=\left(\begin{array}{c}
o(1) \\
s_{22}(k, 0)+o(1)
\end{array}\right) \text { as } t \rightarrow \infty \text { for } k \in N_{5}, \quad \operatorname{Im} k^{3}<0,
\end{aligned}
$$

where $r_{12}=r_{12}\left(\omega^{2} k\right)$ for $k \in N_{1}$ and $r_{12}=r_{12}(\omega k)$ for $k \in N_{3}$.

Substituting (3.27) into (4.5), (4.7) and (4.9), using Propositions 3.2, and 3.4, gives:

$$
\begin{aligned}
& \left(\begin{array}{l}
s_{11}(k, t) \\
s_{21}(k, t)
\end{array}\right)=\left(\begin{array}{c}
s_{11}(k, 0)+o(1) \\
o(1)
\end{array}\right) \text { as } t \rightarrow \infty \text { for } k \in N_{2}, \operatorname{Im} k^{3}>0, \\
& \left(\begin{array}{c}
s_{12}(k, t) \\
s_{22}(k, t)
\end{array}\right)=\left(\begin{array}{c}
o(1) \\
s_{22}(k, 0)-r_{21} s_{12}(k, 0)+o(1)
\end{array}\right) \text { as } t \rightarrow \infty \text { for } k \in N_{4} \cup N_{6}, \quad \operatorname{Im} k^{3}<0,
\end{aligned}
$$

where $r_{21}=r_{21}\left(\omega^{2} k\right)$ for $k \in N_{4}$ and $r_{21}=r_{21}(\omega k)$ for $k \in N_{6}$. 
From the obtained asymptotics it follows that $\tilde{R}(k, t)=o(1), \tilde{r}(k, t)=o(1)$ as $t \rightarrow \infty$. Hence, $R(x+$ $y, t)=o(1)$ as $t \rightarrow \infty$ and for any $x>0$ :

$$
p(x, t)=o(1) \text { as } t \rightarrow \infty .
$$

It is known that if the spectrum of Eq. (2.4) is purely continuous, then the asymptotic solution $p$ as $t \rightarrow \infty$ of the $\mathrm{KdV}$ equation on the whole line is still slowly varying wave train, oscillating about $p=0$, (see [9]). Hence, the asymptotic solution (4.15) at infinity of $t$ of the IBVP (1.1)-(1.3) is different from that of the $\mathrm{KdV}$ equation on the whole line.

\section{Exact soliton-solutions of the Cauchy problem for the KdV equation}

We consider the Cauchy problem for the KdV equation:

$$
p_{t}-6 p p_{x}+p_{x x x}=0, \quad(x, t) \in[0, \infty) \times(-\infty, \infty),
$$

with the known initial condition:

$$
p(x, 0)=p(x)
$$

where $p(x, t)$ is a real-valued function satisfying the condition for any $t \in(-\infty, \infty)$ and some $\varepsilon>0$ :

$$
\int_{0}^{\infty} e^{\varepsilon x}|p(x, t)| \mathrm{d} x<\infty
$$

The Cauchy problem (5.1)-(5.2) is associated with the SP for the Schrödinger equation:

$$
-y_{x x}+p(x, t) y=\rho^{2} y, \quad(x, t) \in[0, \infty) \times(-\infty, \infty)
$$

with the boundary condition:

$$
y(\rho, 0 ; t)=0,
$$

where the potential $p(x, t)$ is a real-valued function satisfying the condition (5.3).

\subsection{The direct and inverse $S P$ (5.4)-(5.5)}

The SP (5.4)-(5.5) with the potential $p(x)$ satisfying condition (5.3) has been investigated in the works $[6,7]$. In the subsection 5.1 we recall the known results of this SP from these works and omit the proof.

Eq. (5.4) with the potential $p(x)$ satisfying condition (5.3) has a solution $e(\rho, x)$, which for each $x \geq 0$ is a holomorphic function of $\rho$ when $\operatorname{Im} \rho>-\frac{\varepsilon}{2}$ and satisfies the asymptotic condition as $x \rightarrow \infty$ :

$$
e(\rho, x)=e^{i \rho x}[1+o(1)], e_{x}^{\prime}(\rho, x)=e^{i \rho x}[i \rho+o(1)] .
$$

For each $\rho_{0}>0$ Eq. (5.4) has a solution $e_{1}(\rho, x)$, which for each $x \geq 0$ is holomorphic function of $\rho$ in the domain $|\rho|>\rho_{0}, \operatorname{Im} \rho>0$, and satisfies the asymptotic condition as $x \rightarrow \infty$ :

$$
e_{1}(\rho, x)=e^{-i \rho x}[1+o(1)], \quad e_{1 x}^{\prime}(\rho, x)=e^{-i \rho x}[-i \rho+o(1)] .
$$

uniformly in $\rho$ in the domain $|\rho|>\rho_{0}>0$.

The functions $e(\rho, x), e(-\rho, x)$ and $e(\rho, x), e_{1}(\rho, x)$ form the fundamental systems of solutions of 
Eq. (5.4) and their Wronskians are equal to:

$$
\begin{aligned}
& W[e(\rho, x), e(-\rho, x)]=-2 i \rho \text { for }|\operatorname{Im} \rho|<\frac{\varepsilon}{2}, \\
& W\left[e(\rho, x), e_{1}(\rho, x)\right]=-2 i \rho \text { for }|\rho|>\rho_{0}, \operatorname{Im} \rho>0, \rho_{0}>0 .
\end{aligned}
$$

The solutions of Eq. (5.4) can be represented in the form:

$$
e(\rho, x)=e^{i \rho x}+\int_{x}^{\infty} K(x, \xi) e^{i \rho \xi} \mathrm{d} \xi
$$

where the kernel $K(x, \xi)$ has first-order continuous partial derivatives with respect to $x$ and $\xi$. Denote by $\omega(\rho, x)$ the solution of the eigenvalue problem generated by Eq. (5.4):

$$
-\omega_{x x}(\rho, x)+p(x) \omega(\rho, x)=\rho^{2} \omega(\rho, x), \quad x \in[0, \infty)
$$

with the initial conditions:

$$
\omega(\rho, 0)=0, \quad \omega_{x}(\rho, 0)=1 .
$$

By virtue of (5.8) and (5.9), the solution of the problem (5.11)-(5.12) is represented in the form:

$$
\begin{aligned}
& \omega(\rho, x)=\frac{e(-\rho) e(\rho, x)-e(\rho) e(-\rho, x)}{2 i \rho} \text { for }|\operatorname{Im} \rho|<\frac{\varepsilon}{2}, \\
& \omega(\rho, x)=\frac{e_{1}(\rho) e(\rho, x)-e(\rho) e_{1}(\rho, x)}{2 i \rho} \text { for }|\rho|>\rho_{0}, \rho>0,
\end{aligned}
$$

where $e(\rho)=e(\rho, 0)$ and $e_{1}(\rho)=e_{1}(\rho, 0)$.

Differentiating the equality (5.14) with respect to $x$, using the initial condition (5.12), we have:

$$
e_{1}(\rho)=\frac{2 i \rho+e(\rho) e_{1 x}^{\prime}(\rho)}{e_{x}^{\prime}(\rho)} \text { for }|\rho|>\rho_{0}, \rho>0 .
$$

By $L$ we mean the operator generated in the space $L_{2}[0, \infty)$ by Eq. (5.4) and boundary condition (5.5). The potential $p_{(x)}$ in the operator $L$ is a real-valued function. Consider an eigenfunction $\Omega(\rho, x)$ of the operator $L$ normalized in the following way:

$$
\Omega(\rho, x)=\frac{2 i \rho \omega(\rho, x)}{e(\rho)}, \operatorname{Im} \rho>-\frac{\varepsilon}{2} .
$$

By (5.13) and (5.14), the normalized eigenfunction $\Omega(\rho, x)$ is represented in the form:

$$
\begin{aligned}
& \Omega(\rho, x)=S(\rho) e(\rho, x)-e(-\rho, x) \text { for }|\operatorname{Im} \rho|<\frac{\varepsilon}{2}, \\
& \Omega(\rho, x)=S_{1}(\rho) e(\rho, x)-e_{1}(\rho, x) \text { for }|\rho|>\rho_{0},
\end{aligned}
$$

where

$$
\begin{aligned}
& S(\rho)=\frac{e(-\rho)}{e(\rho)} \text { for }|\operatorname{Im} \rho|<\frac{\varepsilon}{2} \\
& S_{1}(\rho)=\frac{e_{1}(\rho)}{e(\rho)} \text { for }|\rho|>\rho_{0}, \operatorname{Im} \rho>0 .
\end{aligned}
$$

The functions $S(\rho)$ and $S_{1}(\rho)$ are called the scattering function and the reflection coefficient of the operator $L$, respectively. 
Since the potential in Eq. (5.11) is a real-valued function satisfying estimate (5.3), then all the zeros $\rho_{j}$ of the function $e(\rho)$ are simple and lie on the imaginary axis, i.e., $\rho_{j}=i \mu_{j}, \mu_{j}>\varepsilon_{0}>0, j=$ $1, \ldots, N$. By virtue of this fact, using the expression (5.15), we calculate:

$$
\begin{aligned}
f_{j}(x) & =\left.i \operatorname{Res}\right|_{\rho=i \mu_{j}}\left\{\frac{e_{1}(\rho)}{e(\rho)} e^{i \rho x}\right\} \\
& =\left.i \operatorname{Res}\right|_{\rho=i \mu_{j}}\left\{\frac{2 i \rho+e(\rho) e_{1 x}^{\prime}(\rho)}{e(\rho) e_{x}^{\prime}(\rho)} e^{i \rho x}\right\} \\
& =\left.i \operatorname{Res}\right|_{i \mu_{j}}\left\{\frac{2 i \rho e^{i \rho x}}{e(\rho) e_{x}^{\prime}(\rho)}\right\} \\
& =i \frac{2 i\left(i \mu_{j}\right) e^{-\mu_{j} x}}{e_{\rho}^{\prime}\left(i \mu_{j}\right) e_{x}^{\prime}\left(i \mu_{j}\right)}
\end{aligned}
$$

where $e^{\mu_{j} x} f_{j}(x)$ is expressed through the square of norm $m_{j}^{-62}$ of the Jost solution $e\left(i \mu_{j}, x\right)$ in $L_{2}[0, \infty),[7]:$

$$
e^{\mu_{j} x} f_{j}(x)=-\frac{2 i \mu_{j}}{e_{\rho}^{\prime}\left(i \mu_{j}\right) e_{x}^{\prime}\left(i \mu_{j}\right)}=\left(\int_{0}^{\infty}\left|e\left(i \mu_{j}, x\right)\right|^{2} \mathrm{~d} x\right)^{-1}=m_{j}^{2}>0, \quad j=1, \ldots, N .
$$

We introduce the function:

$$
F_{S}(x)=\frac{1}{2 \pi} \int_{-\infty+i \eta}^{+\infty+i \eta}[S(\rho)-1] e^{i \rho x} \mathrm{~d} \rho,
$$

where $\eta$ is a number satisfying the equality: $0<\eta<\varepsilon_{0}$.

The integral (5.20) is applied to analytic function $S(\rho)-1$ in the strip $0<|\operatorname{Im} \rho|<\varepsilon_{0}$, therefore its value will not depend on $\eta$.

The function $F_{S}(x)$ like the scattering function $S(\rho)$, is a spectral characteristic of the operator $L$ on its continuous spectrum. While the functions $f_{j}(x)$ defined by (5.18) characterize the operator $L$ on its point spectrum.

The scattering function $S(\rho)$, the nonsingular numbers $i \mu_{j}, \ldots, i \mu_{N}$ and the normalization multipliers $m_{1}^{2}, \ldots, m_{N}^{2}$ are called the scattering data of the operator $L$. The scattering data are not independent of each other. The scattering data uniquely determine the self-adjoint operator $L$. To reconstruct this operator from the scattering data, we construct the function [7]:

$$
F(x)=F_{S}(x)+\sum_{j=1}^{N} f_{j}(x)=F_{S}(x)+\sum_{j=1}^{N} m_{j}^{2} e^{-\mu_{j} x} .
$$

The kernel $K(x, y)$ from (5.10) satisfies the GLM equation:

$$
F(x+y)+K(x, y)+\int_{x}^{\infty} K(x, \xi) F(y+\xi) \mathrm{d} \xi=0,0 \leq x<y<\infty .
$$

Eq. (5.22) has a unique solution $K(x, y)$, and the potential $p(x)$ is recovered through the found solution by the equality [7]:

$$
p(x)=-2 \frac{\mathrm{d}}{\mathrm{d} x} K(x, x), \quad x \geq 0 .
$$

where the reconstructed function (5.23) is real-valued and satisfies the same estimate (5.3), as the estimate for the potential in the Schrödinger equation (5.4). 


\subsection{Non-scattering potentials}

There exists a remarkable class of potentials, for which Eq. (5.22) can be solved exactly. These are non-scattering potentials on the half-line, for which the inverse Fourier transform $F_{S}(x)$ defined by (5.20) in the sense of generalized functions is equal to zero, [12]. Hence, in the class of nonscattering potentials the functions $F_{S}(x)$ and $F(x)$ defined by (5.20) and (5.21), respectively, are

$$
F_{S}(x)=0, \quad F(x)=\sum_{j=1}^{N} m_{j}^{2} e^{-\mu_{j} x} .
$$

Our definition of non-scattering potential is similar to the definition of reflectionless of potentials, for which the reflection coefficient is identically zero [8].

Under the condition (5.24) Eq. (5.22) can be solved exactly. Indeed, the solution $K(x, y)$ of this equation is to be sought in the form:

$$
K(x, y)=\sum_{j=1}^{N} K_{j}(x) e^{-\mu_{j} y}, 0 \leq x \leq y, \mu_{j}>\varepsilon_{0}, j=1, \ldots, N .
$$

Substituting (5.25) into Eq. (5.22), after some simple transformations, we obtain a system of linear algebraic equations for $K_{j}(x)$ :

$$
K_{j}(x)+f_{j}(x) \sum_{n=1}^{N} \frac{e^{-\mu_{n} x}}{\mu_{n}+\mu_{j}} K_{n}(x)=-f_{j}(x), \quad j=1, \ldots, N
$$

Let $D(x)$ denote an $N \times N$ square matrix consisting of the elements:

$$
D_{j n}(x)=\delta_{j n}+f_{j}(x) \frac{e^{-\mu_{n} x}}{\mu_{n}+\mu_{j}}, j, n=1, \ldots, N .
$$

From linear algebra, we know that the solution of the system (5.26) is

$$
K_{j}(x)=\frac{\operatorname{det} D^{(j)}(x)}{\operatorname{det} D(x)}, j=1, \ldots, N .
$$

where $D^{(j)}(x)$ stands for the matrix obtained from the matrix $D(x)$ on substituting the elements in its $j$-th column by the elements $-f_{n}(x)$ :

$$
D_{n j}^{(j)}(x)=-f_{n}(x)=-m_{n}^{2} e^{-\mu_{n} x}, n=1, \ldots, N .
$$

Since the potential $p(x)$ is determined by $K(x, x)$, then we calculate it with the help of (5.25):

$$
K(x, x)=(\operatorname{det} D(x))^{-1} \sum_{j=1}^{N} \operatorname{det} D^{(j)}(x) e^{-\mu_{j} x}, \quad x \geq 0 .
$$

Using the rule of differentiation of determinants, we find that the numerator in this expression is equal to the derivative of $\operatorname{det} D(x)$, because $K(x, x)=\frac{\mathrm{d}}{\mathrm{d} x} \ln \operatorname{det} D(x)$. Hence, the formula (5.23) for the potential $p(x)$ in the class of non-scattering potentials is written in a compact form:

$$
p(x)=-2 \frac{\mathrm{d}^{2}}{\mathrm{~d} x^{2}} \ln \operatorname{det} D(x), \quad x \geq 0 .
$$

The expression (5.28) completely describes the whole family of non-scattering potentials. 
P.L. Vu / The description of reflection coefficients of the scattering problems ...

\subsection{The time-dependence of the reflection coefficient}

It is known that the $\mathrm{KdV}$ equation (5.1) is identical to the equation defined by the Lax representation [8]:

$$
\dot{L}=[L, A]=L A-A L,
$$

where $L=-\frac{\mathrm{d}^{2}}{\mathrm{~d} x^{2}}+p, A=4 \frac{\mathrm{d}^{3}}{\mathrm{~d} x^{3}}-3 p_{x}-6 p \frac{\mathrm{d}}{\mathrm{d} x}-\gamma, \dot{L}$ is derivative of $L(t)$ with respect to $t$, and $\gamma$ is some constant, which will be determined below.

The potential $p(x, t)$ in the operator $L(t)$ is called isospectral if the spectrum of $L(t)$ is invariant with $t$, i.e., $\dot{\lambda}=0$. The Lax pair for the KdV equation (5.1) consists of the operator $L(t)$ for the spectral problem and the operator $A$ governing the time-dependence of eigenfunctions. Namely,

$$
\begin{aligned}
& L \Omega=\lambda \Omega, \quad \lambda=\rho^{2}, \quad \operatorname{Im} \rho>-\frac{\varepsilon}{2}, \\
& \dot{\Omega}=-A \Omega, \quad(x, t) \in[0, \infty) \times(-\infty, \infty),
\end{aligned}
$$

where $\Omega$ is the normalized eigenfunction defined by (5.16).

Differentiating Eq. (5.30) with respect to $t$ and using (5.31), we have:

$$
\dot{L} \Omega+L \dot{\Omega}=\dot{L} \Omega-L A \Omega=\dot{\lambda} \Omega+\lambda \dot{\Omega}=\dot{\lambda} \Omega-\lambda A \Omega, \quad\{\dot{L}-(L A-A L)\} \Omega=\dot{\lambda} \Omega .
$$

It follows from (5.32) that the Lax representation (5.29) for the nontrivial eigenfunction $\Omega$ holds if and only if $\dot{\lambda}=0$.

Lemma 5.1. If the potential $p(x, t)$ in the operator $L(t)$ satisfies the $K d V$ equation (5.1), then the time-dependence of the normalization eigenfunction (5.16) is defined by the evolution equation:

$$
\dot{\Omega}=\left(4 i \rho^{3}-4 \frac{\mathrm{d}^{3}}{\mathrm{~d} x^{3}}+6 p \frac{\mathrm{d}}{\mathrm{d} x}+3 p_{x}\right) \Omega, \quad \operatorname{Im} \rho>-\frac{\varepsilon}{2}, \quad(x, t) \in[0, \infty) \times(-\infty, \infty)
$$

and the reflection coefficient $S_{1}(\rho ; t)$ evolves according to the equation:

$$
S_{1}(\rho ; t)=\frac{e_{1}(\rho ; t)}{e(\rho ; t)}=\frac{e_{1}(\rho)}{e(\rho)} e^{8 i \rho^{3} t} \text { for }|\rho|>\rho_{0}, \operatorname{Im} \rho>0, t \in(-\infty, \infty) .
$$

Proof. Let the potential $p(x, t)$ in $L(t)$ satisfy the $\mathrm{KdV}$ equation (5.1), then the time-dependence of the normalized eigenfunction (5.16) is given by the evolution equation (5.31). Using (5.29), we write Eq. (5.31) in the form:

$$
\dot{\Omega}=\left(\gamma-p_{x}\right) \Omega+(4 \lambda+2 p) \Omega_{x}, \lambda=\rho^{2} .
$$

Due to (5.6) and (5.7) the normalization eigenfunction (5.16) obeys the asymptotic condition as $x \rightarrow \infty$ :

$$
\Omega(\rho, x ; t)=\frac{e_{1}(\rho ; t)}{e(\rho ; t)} e^{i \rho x}-e^{-i \rho x}+o(1) \text { for }|\rho|>\rho_{0}>0, \operatorname{Im} \rho>0 .
$$

Since $p(x, t)$ is a solution of the $\mathrm{KdV}$ equation $(5.1)$, then the potential $p(x, t)$ is a isospectral potential. Using this fact and (5.3), (5.36), in (5.35) letting $x$ tend to $\infty$, we find

$$
\frac{\mathrm{d}}{\mathrm{d} t}\left(\frac{e_{1}(\rho ; t)}{e(\rho ; t)}\right) e^{i \rho x}=\gamma\left(\frac{e_{1}(\rho ; t)}{e(\rho ; t)} e^{i \rho x}-e^{-i \rho x}\right)+4 i \rho^{3}\left(\frac{e_{1}(\rho ; t)}{e(\rho ; t)} e^{i \rho x}+e^{-i \rho x}\right),
$$


whence, it follows that for $|\rho|>\rho_{0}>0, \operatorname{Im} \rho>0$ :

$$
\begin{aligned}
-\gamma e^{-i \rho x}+4 i \rho^{3} e^{-i \rho x} & =0, \\
\frac{\mathrm{d}}{\mathrm{d} t}\left(\frac{e_{1}(\rho ; t)}{e(\rho ; t)}\right) e^{i \rho x} & =\left(\gamma+4 i \rho^{3}\right) \frac{e_{1}(\rho ; t)}{e(\rho ; t)} e^{i \rho x} .
\end{aligned}
$$

Hence, $\gamma=4 i \rho^{3}$, and the time-dependence of the functions $\Omega$ and $S_{1}$ defined by evolution equations (5.33) and (5.34) are deduced from (5.31) and (5.37), respectively. The lemma is proved.

The Lemma 5.1 enables us to find the time-dependent potential $p(x, t)$ in the class of nonscattering potentials. In fact, the time-dependent matrix $D(x ; t)$ is obtained from the matrix $D(x)$ given by (5.27) with the help of the following substitution:

$$
D_{j n}(x)=\delta_{j n}+f_{j}(x) \frac{e^{-\mu_{n} x}}{\mu_{n}+\mu_{j}} \rightarrow D_{j n}(x ; t)=\delta_{j n}+f_{j}(x ; t) \frac{e^{-\mu_{n} x}}{\mu_{n}+\mu_{j}},
$$

where $f_{j}(x, t)$ is calculated by the formulas (5.34), (5.18) and (5.19):

$$
\begin{aligned}
f_{j}(x ; t) & =\left.i \operatorname{Res}\right|_{\rho=i \mu_{j}}\left\{S_{1}(\rho ; t) e^{i \rho x}\right\}=\left.i \operatorname{Res}\right|_{\rho=i \mu_{j}}\left\{S_{1}(\rho) e^{8 i \rho^{3} t+i \rho x}\right\} \\
& =\frac{2 \mu_{j}}{i e_{\rho}^{\prime}\left(i \mu_{j}\right) e_{x}^{\prime}\left(i \mu_{j}\right)} e^{-\mu_{j} x+8 \mu_{j}^{3} t}=m_{j}^{2} e^{-\mu_{j} x+8 \mu_{j}^{3} t}=f_{j}(x) e^{8 \mu_{j}^{3} t}
\end{aligned}
$$

in addition $f_{j}(x, 0)=f_{j}(x), j=1, \ldots, N, S_{1}(\rho ; 0)=S_{1}(\rho),(x, t) \in[0, \infty) \times(-\infty, \infty)$.

The formulas (5.28) and (5.38) give exact soliton-solutions of the KdV equation (5.1) in the class of non-scattering potentials:

$$
p(x, t)=-2 \frac{\mathrm{d}^{2}}{\mathrm{~d} x^{2}} \ln \operatorname{det} D(x ; t)
$$

where

$$
D_{j n}(x, t)=\delta_{j n}+\frac{2 \mu_{j}}{i\left(\mu_{j}+\mu_{n}\right) e_{\rho}^{\prime}\left(i \mu_{j}\right) e_{x}^{\prime}\left(i \mu_{j}\right)} e^{-\left(\mu_{j}+\mu_{n}\right) x+8 \mu_{j}^{3} t}, j, n=1, \ldots, N .
$$

The soliton-solution (5.39) of the KdV equation (5.1) is constructed from the non-scattering data $s$ of the associated scattering problem (5.4)-(5.5):

$$
s=\left\{S(\rho ; t) \equiv 1 \text { for }|\operatorname{Im} \rho|<\frac{\varepsilon_{0}}{2}, \rho_{j}=i \mu_{j}, \mu_{j}>\varepsilon_{0}>0, m_{j}^{2}>0, j=1, \ldots, N\right\} .
$$

The non-scattering data (5.41) are formulated from the known initial condition $p(x)=p(x, 0)$ of the Cauchy problem for the $\mathrm{KdV}$ equation (5.1) considered in the class of non-scattering potentials.

Theorem 5.1. Let the function $p(x)$ in the operator $L$ be an isospectral non-scattering potential which is a real-valued continuous function satisfying the estimate (5.3). Then the normalization multipliers $m_{j}^{2}$ are defined by formula (5.19), and the time-dependence of the normalization eigenfunction and the reflection coefficient is defined by formulas (5.33) and (5.34), respectively. By these formulas, the non-scattering potential (5.39) constructed from the given non-scattering data (5.41) describes the whole family of non-scattering potentials which are soliton-solutions of the Cauchy problem for the KdV equation (5.1) with the known initial condition $p(x)$. 


\subsection{An example}

Example. Let the non-scattering data (5.41) consist of two simple poles $\rho_{1}=i \mu_{1}$ and $\rho_{2}=i \mu_{2}$, $\mu_{1}>\mu_{2}>0$. In this case the elements $D_{j n}(x, t)$ of the matrix $D(x, t)$ are calculated by (5.39) and (5.40):

$$
\begin{aligned}
D_{11}(x, t) & =1+\frac{m_{1}^{2}}{2 \mu_{1}} e^{-2 \mu_{1} x+8 \mu_{1}^{3} t}, & D_{12}(x, t) & =\frac{m_{1}^{2}}{\mu_{1}+\mu_{2}} e^{-\left(\mu_{1}+\mu_{2}\right) x+8 \mu_{1}^{3} t}, \\
D_{21}(x, t) & =\frac{m_{2}^{2}}{\mu_{1}+\mu_{2}} e^{-\left(\mu_{1}+\mu_{2}\right) x+8 \mu_{2}^{3} t}, & D_{22}(x, t) & =1+\frac{m_{2}^{2}}{2 \mu_{2}} e^{-2 \mu_{2} x+8 \mu_{2}^{3} t}
\end{aligned}
$$

where $m_{1}^{2}$ and $m_{2}^{2}$ are defined by the formula (5.19).

Putting

$$
\begin{aligned}
& \xi=\mu_{1} x-4 \mu_{1}^{3} t+\xi_{0}, \eta=\mu_{2} x-4 \mu_{2}^{3} t+\eta_{0}, \\
& \xi_{0}=\frac{1}{2} \ln \left(\frac{2 \mu_{1}}{m_{1}^{2}} \frac{\mu_{1}+\mu_{2}}{\mu_{1}-\mu_{2}}\right), \quad \eta_{0}=\frac{1}{2} \ln \left(\frac{2 \mu_{2}}{m_{2}^{2}} \frac{\mu_{1}+\mu_{2}}{\mu_{1}-\mu_{2}}\right) .
\end{aligned}
$$

and using (5.42), we calculate the determinant $D$ of the matrix $D(x, t)$ :

$$
D=\operatorname{det} D(x, t)=1+\frac{\mu_{1}+\mu_{2}}{\mu_{1}-\mu_{2}} e_{1}+\frac{\mu_{1}+\mu_{2}}{\mu_{1}-\mu_{2}} e_{2}+e_{1} e_{2},
$$

where

$$
\begin{aligned}
& e_{1}=e^{-2 \xi}=\frac{m_{1}^{2}}{2 \mu_{1}} \cdot \frac{\mu_{1}-\mu_{2}}{\mu_{1}+\mu_{2}} e^{-2 \mu_{1} x+8 \mu_{1}^{3} t} \\
& e_{2}=e^{-2 \eta}=\frac{m_{2}^{2}}{2 \mu_{2}} \cdot \frac{\mu_{1}-\mu_{2}}{\mu_{1}+\mu_{2}} e^{-2 \mu_{2} x+8 \mu_{2}^{3} t}
\end{aligned}
$$

The non-scattering real-valued potential $p(x, t)$ is calculated by formulas (5.39)-(5.40)

$$
p(x, t)=-2 \frac{\mathrm{d}^{2}}{\mathrm{~d} x^{2}} \operatorname{det} D(x, t)=-2 \frac{D D_{x x}-\left(D_{x}\right)^{2}}{D^{2}}, \quad(x, t) \in[0, \infty) \times(-\infty, \infty)
$$

where the first and the second partial derivatives $D_{x}$ and $D_{x x}$ of the determinant $D$ are found from (5.45), using (5.46):

$$
\begin{aligned}
& D_{x}=-2 \mu_{1} A_{12} e_{1}-2 \mu_{2} A_{12} e_{2}-2\left(\mu_{1}+\mu_{2}\right) e_{1} e_{2} \\
& D_{x x}=4 \mu_{1}^{2} A_{12} e_{1}+4 \mu_{2}^{2} A_{12} e_{2}+4\left(\mu_{1}+\mu_{2}\right)^{2} e_{1} e_{2}, \\
& A_{12}=\frac{\mu_{1}+\mu_{2}}{\mu_{1}-\mu_{2}} .
\end{aligned}
$$


It is easy to verify that

$$
\begin{aligned}
D & =1+A_{12} e^{-2 \xi}+A_{12} e^{-2 \eta}+e^{-2 \xi-2 \eta} \\
& =\frac{e^{-\xi-\eta}}{\mu_{1}-\mu_{2}}\left\{\left(\mu_{1}-\mu_{2}\right) e^{\xi+\eta}+\left(\mu_{1}+\mu_{2}\right) e^{-\xi+\eta}+\left(\mu_{1}+\mu_{2}\right) e^{\xi-\eta}+\left(\mu_{1}-\mu_{2}\right) e^{-\xi-\eta}\right\} \\
& =\frac{e^{-\xi-\eta}}{\mu_{1}-\mu_{2}}\left\{\mu_{1}\left(e^{\xi}+e^{-\xi}\right)\left(e^{\eta}+e^{-\eta}\right)-\mu_{2}\left(e^{\xi}-e^{-\xi}\right)\left(e^{\eta}-e^{-\eta}\right)\right\} \\
& =\frac{e^{-\eta}\left(e^{\eta}+e^{-\eta}\right) e^{-\xi}\left(e^{\xi}-e^{-\xi}\right)}{\mu_{1}-\mu_{2}}\left\{\mu_{1} \frac{\left(e^{\xi}+e^{-\xi}\right)}{\left(e^{\xi}-e^{-\xi}\right)}-\mu_{2} \frac{\left(e^{\eta}-e^{-\eta}\right)}{\left(e^{\eta}+e^{-\eta}\right)}\right\} \\
& =\frac{\left(1+e^{-2 \eta}\right)\left(1-e^{-2 \xi}\right)}{\mu_{1}-\mu_{2}}\left(\mu_{1} \operatorname{cth} \xi-\mu_{2} \operatorname{th} \eta\right) .
\end{aligned}
$$

Hence,

$$
D^{2}=\frac{\left(1+e_{2}\right)^{2}\left(1-e_{1}\right)^{2}}{\left(\mu_{1}-\mu_{2}\right)^{2}}\left(\mu_{1} \operatorname{cth} \xi-\mu_{2} \text { th } \eta\right)^{2} .
$$

Using (5.45), (5.46) and (5.48), we calculate:

$$
\begin{aligned}
& D D_{x x}-D_{x}^{2}=\left(1+A_{12} e_{1}+A_{12} e_{2}+e_{1} e_{2}\right) \times\left(4 \mu_{1}^{2} A_{12} e_{1}+4 \mu_{2}^{2} A_{12} e_{2}+4\left(\mu_{1}+\mu_{2}\right)^{2} e_{1} e_{2}\right)- \\
& 4 \mu_{1}^{2} A_{12}^{2} e_{1}^{2}-4 \mu_{2}^{2} A_{12}^{2} e_{2}^{2}-4\left(\mu_{1}+\mu_{2}\right)^{2} e_{1}^{2} e_{2}^{2}-8 \mu_{1}\left(\mu_{1}+\mu_{2}\right) A_{12} e_{1}^{2} e_{2} \\
& -8 \mu_{2}\left(\mu_{1}+\mu_{2}\right) A_{12} e_{1} e_{2}^{2}-8 \mu_{1} \mu_{2} A_{12}^{2} e_{1} e_{2} \\
& =4 \mu_{1}^{2} A_{12} e_{1}+4 \mu_{2}^{2} A_{12} e_{2}+\left[4\left(\mu_{1}+\mu_{2}\right)^{2}+4\left(\mu_{1}^{2}+\mu_{2}^{2}-2 \mu_{1} \mu_{2}\right) A_{12}^{2}\right] e_{1} e_{2}+ \\
& {\left[4\left(\mu_{1}+\mu_{2}\right)^{2}+4 \mu_{1}^{2}-8 \mu_{1}\left(\mu_{1}+\mu_{2}\right)\right] A_{12} e_{1}^{2} e_{2}+\left[4\left(\mu_{1}+\mu_{2}\right)^{2}+4 \mu_{2}^{2}-8 \mu_{2}\left(\mu_{1}+\mu_{2}\right)\right] A_{12} e_{1} e_{2}^{2}} \\
& =4 \mu_{1}^{2} A_{12} e_{1}+4 \mu_{2}^{2} A_{12} e_{2}+8\left(\mu_{1}+\mu_{2}\right)^{2} e_{1} e_{2}+4 \mu_{2}^{2} A_{12} e_{1}^{2} e_{2}+4 \mu_{1}^{2} A_{12} e_{1} e_{2}^{2} \\
& =4 \mu_{1}^{2} A_{12} e_{1}+4 \mu_{2}^{2} A_{12} e_{2}+8\left(\mu_{1}^{2}-\mu_{2}^{2}\right) A_{12} e_{1} e_{2}+4 \mu_{2}^{2} A_{12} e_{1}^{2} e_{2}+4 \mu_{1}^{2} A_{12} e_{1} e_{2}^{2} \\
& =4 \mu_{1}^{2} A_{12} e_{1}\left(1+2 e_{2}+e_{2}^{2}\right)+4 \mu_{2}^{2} A_{12} e_{2}\left(1-2 e_{1}+e_{1}^{2}\right) \\
& =4 \mu_{1}^{2} A_{12} e_{1}\left(1+e_{2}\right)^{2}+4 \mu_{2}^{2} A_{12} e_{2}\left(1-e_{1}\right)^{2} \\
& =A_{12}\left(1+e_{2}\right)^{2}\left(1-e_{1}\right)^{2}\left\{\mu_{1}^{2} \frac{4 e_{1}}{\left(1-e_{1}\right)^{2}}+\mu_{2}^{2} \frac{4 e_{2}}{\left(1+e_{2}\right)^{2}}\right\} \\
& =\frac{\mu_{1}+\mu_{2}}{\mu_{1}-\mu_{2}}\left(1+e_{2}\right)^{2}\left(1-e_{1}\right)^{2}\left\{\mu_{1}^{2} \frac{4}{\left(e^{\xi}-e^{-\xi}\right)^{2}}+\mu_{2}^{2} \frac{4}{\left(e^{\eta}+e^{-\eta}\right)^{2}}\right\}
\end{aligned}
$$

The explicit soliton-solution $p(x, t)$ of the $\mathrm{KdV}$ equation (5.1) with two bound states is obtained from (5.47), using (5.48), (5.49) and (5.50):

$$
p(x, t)=-2\left(\mu_{1}^{2}-\mu_{2}^{2}\right) \frac{\mu_{1}^{2} \operatorname{cosech}^{2} \xi+\mu_{2}^{2} \operatorname{sech}^{2} \eta}{\left(\mu_{1} \operatorname{cth} \xi-\mu_{2} \operatorname{th} \eta\right)^{2}},(x, t) \in[0, \infty) \times(-\infty, \infty),
$$

where $\xi$ and $\eta$ are determined by (5.43) and (5.44), $\mu_{1}>\mu_{2}>0$.

Thus, $p(x, t)$ represents the nonlinear superposition of two forms, one traveling with speed $4 \mu_{1}^{2}$, the other traveling with speed $4 \mu_{2}^{2}$. If $t$ is very large negative, then using (5.45), (5.46) and (5.48) from 
(5.47) we have:

$$
p(x, t)=o(1) \text { as } t \rightarrow-\infty .
$$

We suppose that $\mu_{1}>\mu_{2}$, then from (5.43)-(5.44) it follows that for every $x$ :

$$
\xi-\eta=\left(\mu_{1}-\mu_{2}\right)\left[x-4\left(\mu_{1}^{2}+\mu_{1} \mu_{2}+\mu_{2}^{2}\right) t\right]+\xi_{0}-\eta_{0} \rightarrow \infty \text { as } t \rightarrow-\infty,
$$

i.e., for $t$ much less than zero and for those valued of $x$, where $\xi$ is about one and $\eta$ is very negative, we have

$$
\begin{aligned}
& \text { sech } \eta \rightarrow 0, \text { and th } \eta \rightarrow-1, \text { therefore } \\
& p(x, t) \rightarrow-2\left(\mu_{1}^{2}-\mu_{2}^{2}\right) \frac{\mu_{1}^{2} \operatorname{cosech}^{2} \xi}{\left(\mu_{1} \operatorname{cth} \xi+\mu_{2}\right)^{2}}=-2 \mu_{1}^{2} \operatorname{sech}^{2}(\xi+\Delta)
\end{aligned}
$$

where $\Delta=\frac{1}{2} \ln \left(\frac{\mu_{1}+\mu_{2}}{\mu_{1}-\mu_{2}}\right)$.

While in the region of $x$, where $\eta$ is about one and $\xi$ is very positive, we obtain:

$$
\begin{aligned}
& \xi-\eta \rightarrow \infty \text { as } t \rightarrow-\infty, \text { and } \operatorname{cosech} \xi \rightarrow 0, \operatorname{cth} \xi \rightarrow 1, \\
& p(x, t) \rightarrow-2\left(\mu_{1}^{2}-\mu_{2}^{2}\right) \frac{\mu_{2}^{2} \operatorname{sech}^{2} \eta}{\left(\mu_{1}-\mu_{2} \text { th } \eta\right)^{2}}=-2 \mu_{2}^{2} \operatorname{sech}^{2}(\eta-\Delta) .
\end{aligned}
$$

That is, for very large negative $t$ the solution looks like two solitary pulses, the large one to the left of the small one.

After a long time, when $t$ is large positive, it follows from (5.43)-(5.44) that for every $x$ :

$$
\eta-\xi=\left(\mu_{2}-\mu_{1}\right)\left[x-4\left(\mu_{1}^{2}+\mu_{1} \mu_{2}+\mu_{2}^{2}\right) t\right]+\eta_{0}-\xi_{0} \rightarrow \infty \text { as } t \rightarrow \infty,
$$

i.e., for large positive $t$ and for those values of $x$, where $\eta$ is order one and $\xi$ very large negative, then

$$
\begin{aligned}
& \operatorname{cosech} \xi \rightarrow 0, \text { and } \operatorname{cth} \xi \rightarrow-1 \text { as } \xi \rightarrow-\infty, \text { therefore, } \\
& p(x, t) \rightarrow-2\left(\mu_{1}^{2}-\mu_{2}^{2}\right) \frac{\mu_{2}^{2} \operatorname{sech}^{2} \eta}{\left(\mu_{1}+\mu_{2} t h \eta\right)^{2}}=-2 \mu_{2}^{2} \operatorname{sech}^{2}(\eta+\Delta) .
\end{aligned}
$$

While if $\xi$ is order one, and $\eta$ very large positive, then

$$
\begin{aligned}
& \text { sech } \eta \rightarrow 0, \text { and th } \eta \rightarrow 1, \text { as } \eta \rightarrow \infty, \text { therefore, } \\
& p(x, t) \rightarrow-2\left(\mu_{1}^{2}-\mu_{2}^{2}\right) \frac{\mu_{1}^{2} \operatorname{cosech}^{2} \xi}{\left(\mu_{1} \operatorname{cth} \xi-\mu_{2}\right)^{2}}=-2 \mu_{1}^{2} \operatorname{sech}^{2}(\xi-\Delta)
\end{aligned}
$$

That is after a long time the large solitary pulse is to the right of the small solitary pulse. They have coalesced and reemerged with their shaped unscathed. The only remnant of the interaction is the phase shift $\Delta=\frac{1}{2} \ln \left(\frac{\mu_{1}+\mu_{2}}{\mu_{1}-\mu_{2}}\right)$. That is the large pulse is moved forward by an amount $2 \Delta / \mu_{1}$ relative to where it would have been in the absence of an interaction, and the small pulse is retarded by an amount $2 \Delta / \mu_{2}$ relative to where it would have been in an unperturbed situation. In Figure 3 we show a sketch of this soliton-solution of the KdV equation (5.1) with two bound states at four successive times. 


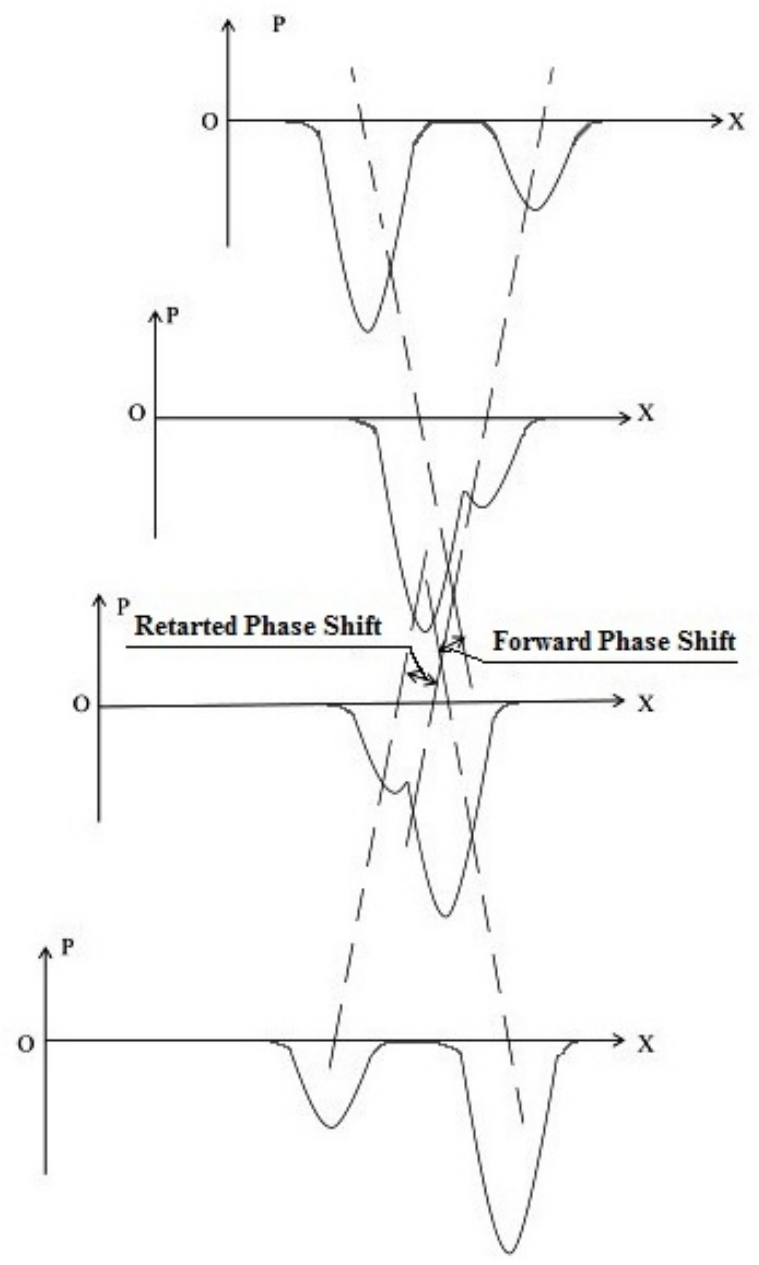

Fig. 3. Two soliton-solution of the KdV equation (5.1) with two bound states at the four successive moments of time $t=t_{0}, t_{1}, t_{2}$ and $t_{3}$.

In general, the non-scattering solution with $N$ bound states has a similar behavior. In this case the non-scattering data (5.41) consist of $N$ simple poles: $\rho_{j}=i \mu_{j}, \mu_{j}>0, j=1, \ldots, N$. With $N$ bound states the solution resembles the superposition of $N$ solitary pulses whose speeds and amplitudes are determined by the positive values $\mu_{j}, j=1, \ldots, N$. The solitary pulses emerge unscathed from interaction except for a phase shift given by the sum of phase shifts from all possible pairwise interactions.

\section{Conclusions}

By Propositions 3.6, 3.7 and formulas (3.42)-(3.44), the self-conjugate matrix (3.5) is found uniquely from the known conditions (1.2) and (1.3). Then the time-dependence of $s(k, t)$ is derived by (4.4)-(4.9). The known function $\tilde{R}(k, t)$ in Eq. (4.10) is defined by ratio (4.11), in which $s_{11}(k ; t)$ and $s_{12}(k ; t)$ for every $t \geq 0$ are any given complex-valued functions satisfying the sufficient conditions of Theorem 2.1. Thus, the application of obtained results to solving the IBVP (1.1)-(1.2)-(1.3) is consistent and is effectively embedded in the ISM schema. 
By Theorem 5.1 the non-scattering potential (5.39) constructed from the non-scattering data (5.41) of the scattering problem (5.4)-(5.5) describes the whole family of non-scattering potentials which are soliton-solutions of the Cauchy problem for the KdV equation (5.1).

\section{Acknowledgments}

This work has been supported by the Vietnam National Foundation for Science and Technology Development (NAFOSTED) under grant number 107.03-2015.31.

\section{References}

[1] A.S. Fokas, Integrable nonlinear evolution equations on the half-line, Communications in Mathematical Physics 230 (1) (2002) 1-39.

[2] A.S. Fokas and A.R. Its, The linearization of the initial-boundary value problem of the nonlinear Schrödinger equation, SIAM Journal on Mathematical Analysis 27 (3) (1996) 738-764.

[3] I.T. Habibullin, Initial boundary value problem for the KdV equation on a semiaxis with homogeneous boundary conditions, Theoretical and Mathematical Physics 130 (2002) 25-44.

[4] I.T. Habibullin, KdV equation on a half-line with the zero boundary condition, Theoretical and Mathematical Physics 119 (3) (1999) 712-718.

[5] B.M. Levitan, Inverse Sturm-Liouville problems (VNU Science Press BC, Utrecht, The Netherlands, 1984).

[6] V.E. Lyantce, An analog of the inverse problem of scattering theory for a nonselfadjoint operator, Mathematics of the USSR-Sbornik 1 (4) (1967) 485-504.

[7] V.A. Marchenko, Sturm-Liouville operators and applications (Springer Basel AG, 1986).

[8] S. Novikov, S.V. Manakov, L.P. Pitaevskii and V.E. Zakharov, Theory of solitons: The inverse scattering method (Springer Science \& Business Media, 1984).

[9] H. Segur, The Korteweg-de Vries equation and water waves. Solutions of the equation. Part 1, Journal of Fluid Mechanics 59 (4) (1973) 721-736.

[10] V.S. Vladimirov, Equations of mathematical physics (Marcel Dekker, Inc., New York, 1971).

[11] P.L. Vu, An initial-boundary value problem for the Korteweg-de Vries equation with dominant surface tension, Acta Applicandae Mathematicae 129 (1) (2014) 41-59.

[12] P.L. Vu, Cauchy problems for a system of non-linear equations and for the non-linear Schrodinger equation, Inverse Problems 10 (2) (1994) 415-429.

[13] P.L. Vu, The initial-boundary value poblem for the Korteweg-de Vries equation on the positive quarterplane, Journal of Nonlinear Mathematical Physics 14 (1) (2007) 28-43.

[14] P.L. Vu, The inverse scattering problem for a system of Dirac equations on a half-line, Selected works "Linear boundary value problems of Mathematical Physics", Institute of Mathematics, National Academy of Sciences of the Ukraine (1973) 174-207 (in Russian). 